\title{
A sand fly salivary protein vaccine shows efficacy against vector-transmitted cutaneous leishmaniasis in nonhuman primates
}

\author{
Fabiano Oliveira, ${ }^{1}$ Edgar Rowton, ${ }^{2}$ Hamide Aslan, ${ }^{1 *}$ Regis Gomes, ${ }^{1,3}$ Philip A. Castrovinci, ${ }^{1}$ \\ Patricia H. Alvarenga, ${ }^{4,5}$ Maha Abdeladhim, ${ }^{1}$ Clarissa Teixeira, ${ }^{1,3}$ Claudio Meneses, ${ }^{1}$ \\ Lindsey T. Kleeman, ${ }^{1}$ Anderson B. Guimarães-Costa, ${ }^{1}$ Tobin E. Rowland, ${ }^{2}$ Dana Gilmore, ${ }^{1}$ \\ Seydou Doumbia, ${ }^{6}$ Steven G. Reed, ${ }^{7}$ Phillip G. Lawyer, ${ }^{2}$ John F. Andersen, ${ }^{8}$ \\ Shaden Kamhawi, ${ }^{1 \dagger}$ Jesus G. Valenzuela ${ }^{1 \dagger}$
}

Currently, there are no commercially available human vaccines against leishmaniasis. In rodents, cellular immunity to salivary proteins of sand fly vectors is associated to protection against leishmaniasis, making them worthy targets for further exploration as vaccines. We demonstrate that nonhuman primates (NHP) exposed to Phlebotomus duboscqi uninfected sand fly bites or immunized with salivary protein PdSP15 are protected against cutaneous leishmaniasis initiated by infected bites. Uninfected sand fly-exposed and 7 of 10 PdSP15-immunized rhesus macaques displayed a significant reduction in disease and parasite burden compared to controls. Protection correlated to the early appearance of Leishmania-specific CD4 ${ }^{+}$IFN- $\gamma^{+}$lymphocytes, suggesting that immunity to saliva or PdSP15 augments the host immune response to the parasites while maintaining minimal pathology. Notably, the $30 \%$ unprotected PdSP15-immunized NHP developed neither immunity to PdSP15 nor an accelerated Leishmaniaspecific immunity. Sera and peripheral blood mononuclear cells from individuals naturally exposed to $P$. duboscqi bites recognized PdSP15, demonstrating its immunogenicity in humans. PdSP15 sequence and structure show no homology to mammalian proteins, further demonstrating its potential as a component of a vaccine for human leishmaniasis.

\section{INTRODUCTION}

Leishmaniasis is a neglected tropical disease that affects the poorest of communities and comes only second to malaria and fourth among tropical parasitic diseases in mortality and morbidity, respectively (1). Despite its global distribution and substantial disease burden, there are no commercially available human leishmaniasis vaccines to date.

All forms of leishmaniasis are transmitted by the bite of infected phlebotomine sand flies. As infected females feed on mammalian hosts, they inject saliva, counteracting hemostasis and improving bloodfeeding success. Leishmania-infected sand flies regurgitate parasites together with the salivary proteins into the bite wound. Exploiting the concurrence of sand fly saliva and parasites in the bite site is an original approach to traditional Leishmania vaccines.

Experimentally, it has been shown that exposure to saliva through bites of uninfected sand flies or immunization with an appropriate salivary protein protects rodents against cutaneous and visceral leishmaniases (2-5). Saliva-mediated protection from leishmaniasis correlates to the induction of a rapid sand fly saliva-specific $\mathrm{T}_{\mathrm{H}} 1$ ( $\mathrm{T}$ helper 1 cell)

\footnotetext{
Vector Molecular Biology Section, Laboratory of Malaria and Vector Research, National Institute of Allergy and Infectious Diseases, National Institutes of Health, Rockville, MD 20852, USA. ${ }^{2}$ Department of Entomology, Walter Reed Army Institute of Research, Silver Spring, MD 20910, USA. ${ }^{3}$ Centro de Pesquisas Gonçalo Moniz (CPqGM)-Fundação Oswaldo Cruz (FIOCRUZ), Salvador, Bahia 40296-710, Brazil. " Laboratório de Bioquímica de Resposta ao Estresse, Instituto de Bioquímica Médica, Universidade Federal do Rio de Janeiro, Rio de Janeiro 21941-902, Brazil. ${ }^{5}$ Instituto Nacional de Ciência e Tecnologia em Entomologia Molecular (INCT-EM), Universidade Federal do Rio de Janeiro, Rio de Janeiro 21941-902, Brazil. ${ }^{6}$ Faculty of Medicine, Pharmacy and Odontostomatology, University of Bamako, Bamako 1805, Mali. 'Infectious Disease Research Institute, Seattle, WA 98102, USA. ${ }^{8}$ Vector Biology Section, Laboratory of Malaria and Vector Research, National Institute of Allergy and Infectious Diseases, National Institutes of Health, Rockville, MD 20852, USA. *Present address: Nursing Department, Faculty of Health Science, Selahaddin Eyyubi University, Diyarbakir, Turkey.

tCorresponding author.E-mail:jvalenzuela@niaid.nih.gov (J.G.V.); skamhawi@niaid.nih.gov (S.K.)
}

immune response at the bite site that steers the development of a faster and more robust Leishmania-specific $\mathrm{T}_{\mathrm{H}} 1$ immunity with minimal pathology $(4,6)$. Moreover, antibodies are not required for saliva-mediated protection from leishmaniasis in murine models $(3,4)$.

Additionally, saliva-driven immunity protected against vector-transmitted leishmaniasis $(3,4)$. This virulent mode of challenge, encompassing sand fly saliva, promastigote secretory gel (7), and midgut-differentiated Leishmania metacyclics, was shown to rescind the efficacy of a vaccine established via needle challenge with Leishmania parasites (8), highlighting the robustness of saliva-mediated immunity to leishmaniasis.

Cutaneous leishmaniasis (CL) is the most widely distributed form of the complex of diseases referred to as the leishmaniases. Annually, an estimated 0.7 million to 1.3 million new CL cases occur worldwide (9). Some twothirds of new CL cases occur in six countries including Afghanistan, Algeria, Brazil, Colombia, Iran, and the Syrian Arab Republic (9). CL caused by Leishmania major is prevalent in the Middle East, North Africa, and SubSaharan Africa, where it is mainly transmitted by Phlebotomus papatasi or Phlebotomus duboscqi sand flies (10). Here, we tested the capacity of exposure to $P$. duboscqi uninfected bites or immunization with its $15-\mathrm{kD}$ salivary protein, PdSP15, in nonhuman primates (NHP) for protection against vector-transmitted $L$. major. We uphold the concept of using immunity to vector salivary proteins to protect humans from CL, demonstrating their efficacy against vector-transmitted L. major in NHP.

\section{RESULTS}

Exposure to uninfected sand fly bites protects NHP against sand fly-transmitted CL

To induce immunity to sand fly saliva in NHP, we exposed naïve rhesus macaques to 20 P. duboscq $i$ uninfected sand fly bites four times every 
21 days. Most NHP (63\%) developed a delayed-type hypersensitivity (DTH) response showing a marked recruitment of mononuclear cells to the dermis 48 hours after the last exposure (Fig. 1A). The reactive bite site showed a relative abundance of interferon- $\gamma$ (IFN- $\gamma$ ) compared to controls ascribing a $\mathrm{T}_{\mathrm{H}} 1$-like environment to the observed cell infiltrate $(P=$ 0.0043, Mann-Whitney test; $n=6$; Fig. 1B). Exposure to uninfected sand flies also generated a humoral response that was more pronounced in DTH-positive reactive animals $(P=0.0002, t$ test; $n=10$; Fig. 1C). To test the efficacy of the observed immunity to uninfected sand fly bites in protection from Leishmania parasites, we developed a natural model of vectortransmitted CL in NHP using L. majorinfected $P$. duboscqi sand flies. Two groups of NHP challenged with either 20 or 50 infected sand flies presented with 50 and $90 \%$ of diseased animals, respectively. Lesion clusters evolved from papules to nodules to ulcerated lesions, which progressed to healed scars (fig. S1), characteristics that mirror those observed in human CL caused by L. major (11). Next, we tested whether immunity generated to sand fly salivary proteins in NHP was protective against vector-transmitted CL. Naïve and uninfected sand fly-exposed NHP were challenged with 50 Leishmaniainfected sand flies. Compared to naïve animals, uninfected sand fly-exposed NHP controlled the infection with a significant reduction in disease burden, as defined by the computation of the cumulative measurement of the largest diameter of each lesion on a weekly basis $(P=0.0083$, Mann-Whitney test; $n=9$ to 15 ; Fig. 1D), maximum lesion size $(P=0.0119$, MannWhitney test; $n=9$ to 15 ; Fig. 1E), and time to heal $[P=0.0048$, log-rank (MantelCox) test; $n=9$ to 15 ; Fig. 1F]. At the 9 -week post-challenge time point, $70 \%$ of naïve animals displayed ulcerated lesions compared to only $30 \%$ of uninfected sand fly-exposed NHP (Fig. 1G). This reduction in disease severity correlated to a significantly lower number of parasites in lesion biopsies $(P=0.0237$, Mann-Whitney test; $n=9$ to 15 ;Fig. $1 \mathrm{H}$ ). Notably, in order not to disrupt the course of lesion development, the parasite burden was measured at 12 weeks after infection when lesions of both naïve and uninfected sand flyexposed NHP were healing. Furthermore,
A
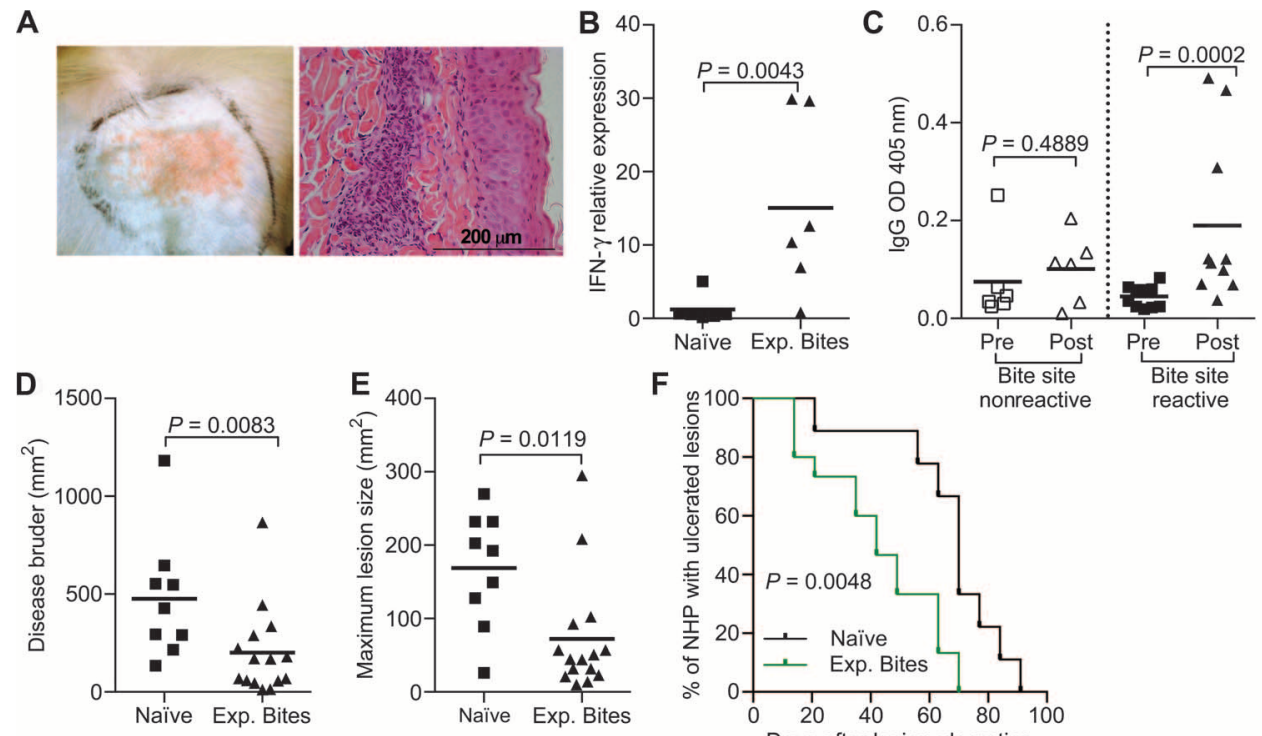

F
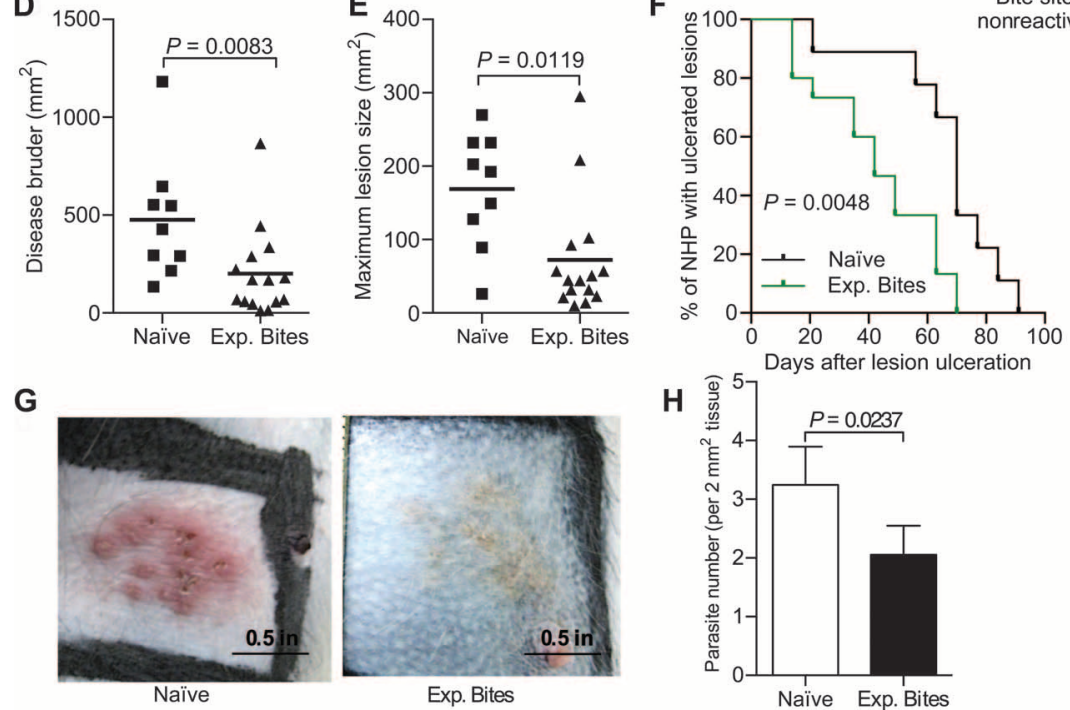

H
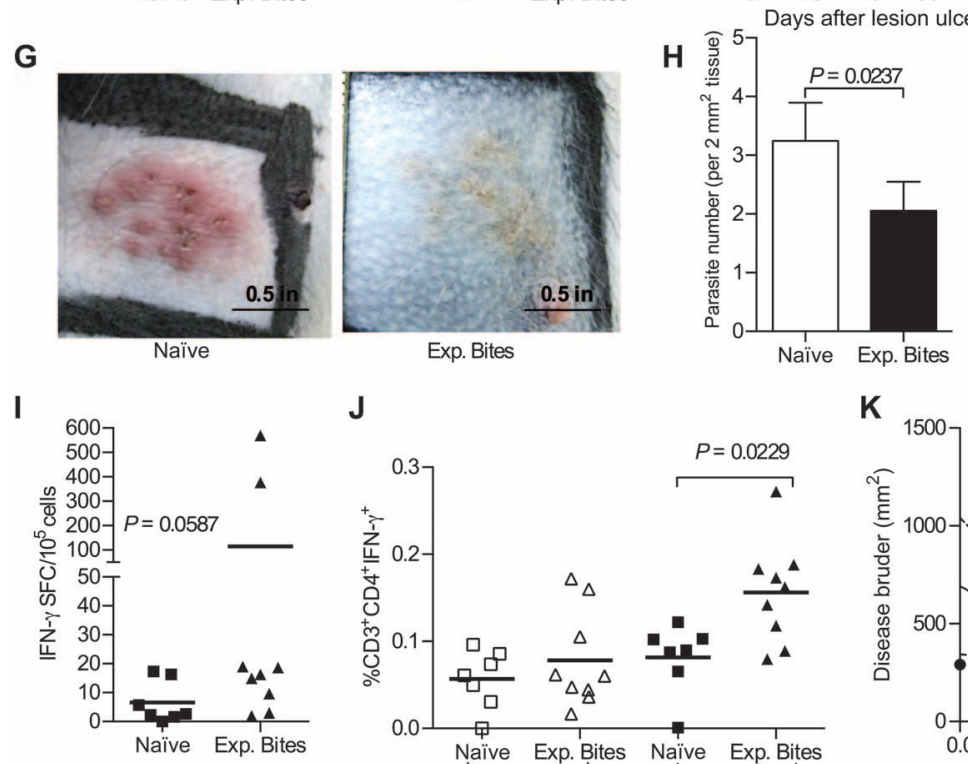

J
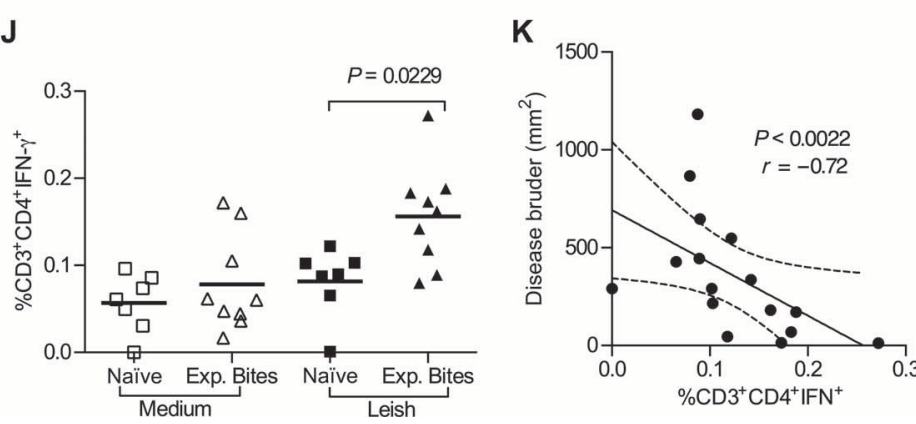

Fig. 1. Exposure to $P$. duboscqi uninfected sand flies (USFs) induces an anti-saliva immunity that protects NHP from vector-transmitted CL. (A to C) Immunity to USF bites 48 hours (A and $B$ ) or 2 weeks (C) after the last exposure. (A) DTH response (left panel) and a hematoxylin and eosin-stained biopsy section (right panel, $\times 400$ ) from a USF bite site. (B) IFN- $\gamma$ mRNA expression in biopsies of a USF bite site (Exp. Bites) or normal skin (Naïve) from the same animal. Biopsies were obtained from six randomly selected bite site-reactive NHP ( $P=0.0043$, Mann-Whitney test; $n=6)$. (C) Anti-saliva immunoglobulin $\mathrm{G}$ (lgG) levels before (Pre) or after (Post) exposure $(P=0.0002, t$ test; $n=10)$. Cumulative data from two independent experiments are shown. OD, optical density. (D to K) Fifteen USF-exposed NHP (Exp. Bites) and nine naïve NHP were challenged with 50 L. major-infected $P$. duboscqi. Cumulative data from two independent experiments are shown. (D) Disease burden ( $P=0.0083$, Mann-Whitney test; $n=9$ to 15). (E) Maximum lesion size ( $P=0.0119$, Mann-Whitney test; $n=9$ to 15). (F) Kaplan-Meier plot of the healing time, a cumulative measurement of lesion development from ulcer to scar $[P=0.0048$, log-rank (MantelCox) test; $n=9$ to 15]. (G) Representative photographs 9 weeks after challenge. (H) Parasite number 12 weeks after challenge ( $P=0.0237$, Mann-Whitney test; $n=9$ to 15 ). (I to K) PBMCs from seven naïve (Naïve) and nine USF-exposed NHP (Exp. Bites) were stimulated with Leishmania antigen (Leish) 2 weeks after challenge. Selection was based on cell number and viability. (I) IFN- $\gamma$ SFC by enzyme-linked immunospot (ELISPOT) ( $P=0.0587$, Mann-Whitney test; $n=7$ to 9). (J) Percent of CD4 ${ }^{+}$IFN- $\gamma^{+}$lymphocytes by flow cytometry ( $P=0.0229$, Mann-Whitney test; $n=7$ to 9 ). (K) Frequency of $\mathrm{CD}^{+}$IFN- $\gamma^{+}$lymphocytes correlated to disease burden. Dashed line indicates $95 \%$ confidence interval $(\mathrm{Cl})(P=0.0022, n=16$, Spearman test $r=-0.72$ ). Scale bar, $200 \mu \mathrm{m}$; lines and bars indicate the mean, and error bars indicate SEM. 
protection in uninfected sand fly-exposed NHP correlated to the induction of an early Leishmania-specific immune response 2 weeks after infection. After stimulation with Leishmania antigen (Leish), peripheral blood mononuclear cells (PBMCs) from uninfected sand flyexposed NHP produced a higher number of IFN- $\gamma$ spot-forming cells (SFC) compared to controls $(P=0.0587$, Mann-Whitney test; $n=7$ to 9; Fig. 1I). We corroborated these data by flow cytometric analysis demonstrating that $\mathrm{CD}^{+}$cells were the main source of specific antiLeishmania IFN- $\gamma$ (fig. S2). Although $\mathrm{CD}^{+}$(Fig. $1 \mathrm{~J}$ ) and $\mathrm{CD} 8^{+}$(fig. S3A) lymphocytes produced Leishmania-specific IFN- $\gamma$, only the frequency of $\mathrm{CD}^{+}{ }^{+} \mathrm{IFN}-\gamma^{+}$cells was statistically higher between uninfected sand fly-exposed and naïve animals $(P=0.0229$, Mann-Whitney test; $n=7$ to 9 ; Fig. 1J). Moreover, the frequency of $\mathrm{CD} 4^{+} \mathrm{IFN}-\gamma^{+}$cells inversely correlated to disease burden in uninfected sand fly-exposed NHP $(P<0.0022, n=16$, Spearman rank correlation test $r=-0.7211$; Fig. $1 \mathrm{~K}$ and fig. S3B), suggesting that Leishmania-specific CD4 T cells are participating in parasite clearance.

\section{Reverse antigen screening of $\boldsymbol{P}$. duboscqi sand fly salivary molecules in saliva-exposed NHP identifies PdSP15 as a vaccine candidate against $\mathrm{CL}$}

Having established that exposure to uninfected sand fly bites protects NHP against CL, our next objective was to identify the salivary protein responsible for the protective effect. Therefore, we screen about 23 secreted salivary proteins of $P$. duboscqi in NHP. To identify protective sand fly salivary proteins while minimizing the number of NHP, we developed an approach named "reverse antigen screening" (RAS). The approach is based on exploiting the adaptive immunity generated against salivary proteins in uninfected sand fly-exposed NHP. Uninfected sand fly-exposed and bite site-reactive NHP were injected intradermally with DNA plasmids coding for the most abundant secreted $P$. duboscqi salivary proteins. Using the host machinery as a natural protein expression system, we selected salivary molecules that induced a $\mathrm{T}_{\mathrm{H}}$ 1-DTH 48 hours after inoculation. Salivary gland homogenate (SGH) and bites from one uninfected sand fly, and empty plasmid were used as positive and negative controls, respectively. From the 23 tested DNA plasmids, we selected the top five molecules based on their induction of the largest skin induration as measured by the diameter of the skin reaction. These included PdMu54 (2.4 mm), PdSP15 (1.83 mm), PdMu29 (1.79 mm), PdMu49 (1.69 mm), and PdMu35 (1.66 mm). We also selected a negative control, empty DNA plasmid $(1.01 \mathrm{~mm})$, and two positive controls, SGH $(3.63 \mathrm{~mm})$ and a bite site $(2.84 \mathrm{~mm})$ (Fig. 2A and table $\mathrm{S} 1$ ). Of the five, $P d S P 15$ was the only molecule displaying a significant increase in IFN- $\gamma$ mRNA message compared to the negative control $[P=0.0109$, one-way analysis of variance (ANOVA); $n=8]$, and the one exhibiting the lowest level of interleukin-4 (IL-4) (Fig. 2B); this translated to a high IFN- $\gamma / \mathrm{IL}-4$ ratio indicative of a $\mathrm{T}_{\mathrm{H}} 1$-biased immune response ( $P=0.0470$, one-way ANOVA; $n=8$; Fig. $2 \mathrm{C})$. Exact $P$ values for the five tested samples are presented in table S2.

\section{Immunization with PdSP15 protects NHP against sand fly-transmitted CL}

NHP were immunized intradermally with PdSP15 DNA two times 21 days apart and boosted 21 days later with recombinant PdSP15 (rPdSP15) and glucopyranosyl lipid adjuvant in stable emulsion (GLA-SE). Control animals were inoculated with empty plasmid followed by a boost with bovine serum albumin and GLA-SE. In contrast to controls, 70\% of PdSP15-immunized NHP displayed a distinct skin induration at the injection site 48 hours after the rPdSP15 boost $(P=0.0067$, MannWhitney test; $n=10$; Fig. 3A). Two weeks later, PBMCs of skin-reactive PdSP15-immunized NHP produced significantly higher IFN- $\gamma$ SFC after stimulation with rPdSP15 compared to controls $(P=0.0002$, MannWhitney test; $n=7$; Fig. 3B, solid squares). The number of IFN- $\gamma$ SFC in the $30 \%$ nonreactive PdSP15-immunized animals was similar to controls (Fig. 3B, empty squares) but produced significantly high levels of specific anti-rPdSP15 IgG antibodies (Fig. 3C, empty squares; $P<$ 0.0001 , one-way ANOVA; $n=3$ ), with antibody levels showing a negative correlation to IFN- $\gamma$ production $(P=0.0037 ; r=-0.84$,
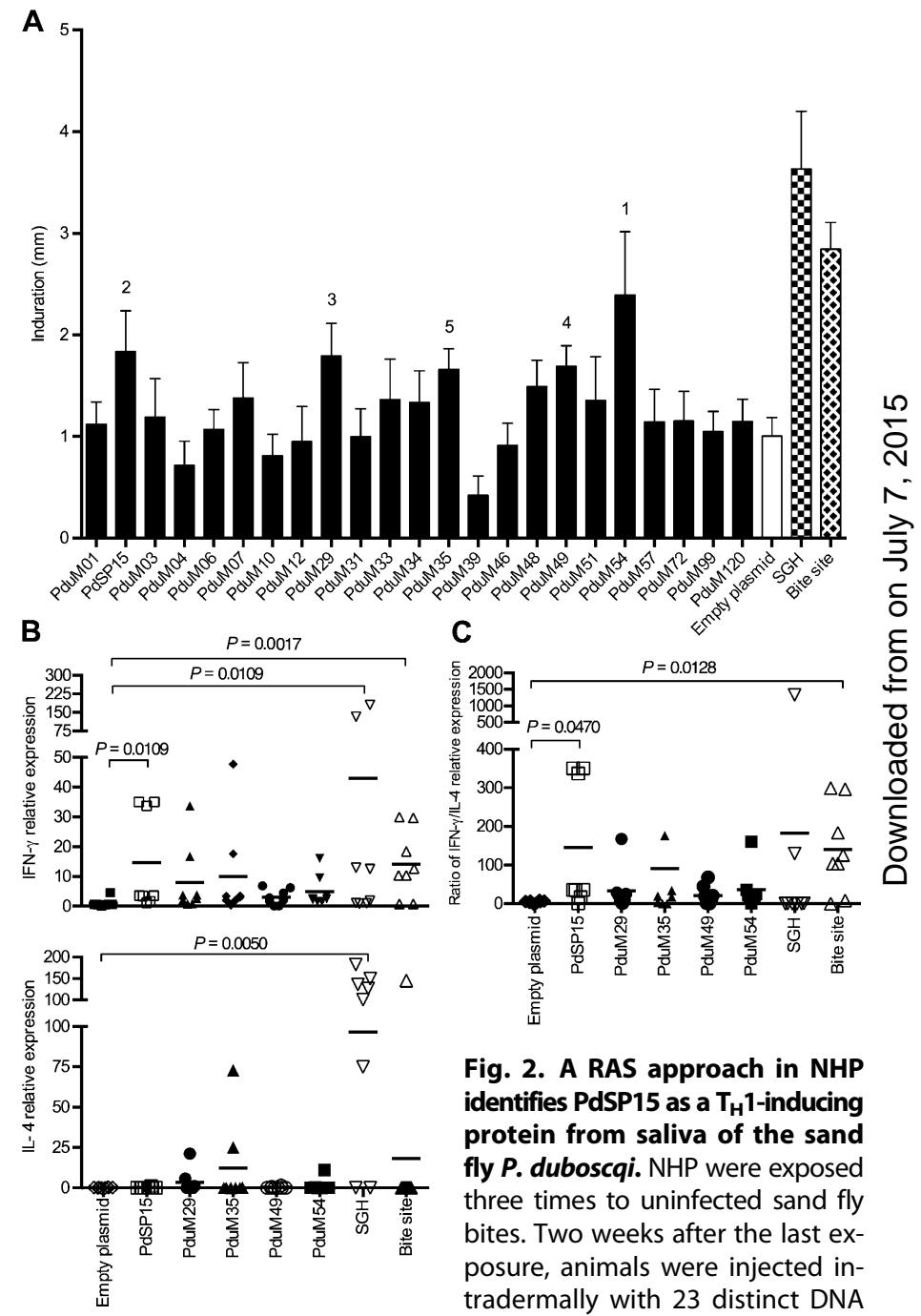

Fig. 2. A RAS approach in NHP identifies PdSP15 as a $T_{H} 1$-inducing protein from saliva of the sand fly $\boldsymbol{P}$. duboscqi. NHP were exposed three times to uninfected sand fly bites. Two weeks after the last exposure, animals were injected intradermally with 23 distinct DNA plasmids encoding the most abundant $P$. duboscai salivary proteins or an empty plasmid as a negative control. Bites from one sand fly or the inoculation of one pair of SGH was used as positive controls. (A) Skin induration 48 hours after inoculation of plasmids measured using a Vernier caliper. Cumulative data of 14 NHP from three independent experiments are shown. (B and $\mathbf{C})$ Two-millimeter skin biopsies of marked injection sites were obtained from 8 of 14 USF-exposed NHP. (B) IFN- $\gamma$ and IL-4 mRNA expression by quantitative real-time fluorescence polymerase chain reaction (RT-qPCR). (C) IFN- $\gamma / \mathrm{IL}-4$ ratio for each animal. Exact measurements and $P$ values for all the samples tested are presented in tables S1 and S2. Lines and bars indicate the mean, and error bars indicate SEM. 
Fig. 3. Immunization with PdSP15 protects NHP against vector-transmitted CL. (A to C) Immunity in PdSP15- immunized (PdSP15) or sham-immunized (CTL) NHP 48 hours ( $A$ ) or 2 weeks ( $B$ and $C$ ) after last immunization. (A) Skin induration after inoculation with bovine serum albumin (CTL) or rPdSP15 ( $P=0.0067, t$ test; $n=10)$. (B) IFN- $\gamma$ SFC by ELISPOT ( $P=0.0002, t$ test; $n=10)$. (C) Anti-saliva lgG levels before (Pre) or after (Post) immunization in controls (CTL), PdSP15immunized NHP producing IFN- $\gamma$ (PdSP15$\left.\mathrm{IFN}^{+}\right)$or not (PdSP15-IFN') $(P<0.0001$, one-way ANOVA; $n=3$ to 10 ). (D to $\mathbf{L}$ ) Evaluation of disease (D to H) and Leishmania-specific immunity ( $($ to $L)$ in $C T L, P d S P 15-I F N^{+}$, or PdSP15-IFN ${ }^{-}$NHP after challenge with 50 infected sand flies. (D) Disease burden $(P=$ 0.0490 , one-way ANOVA; $n=3$ to 11). (E) Maximum lesion size $(P=0.0465$, one-way ANOVA; $n=3$ to 11). (F) Kaplan-Meier plot of the healing time $[P=0.1770$, log-rank (MantelCox) test; $n=3$ to 11]. (G) Representative photographs 5 weeks after challenge. $(\mathrm{H})$ Parasite number 5 weeks after challenge $(P=$ 0.0034 , one-way ANOVA; $n=3$ to 8). (I to K) PBMCs stimulated with Leishmania antigen (Leish) 2 weeks after challenge in 8 to $10 \mathrm{NHP}$. Selection was based on cell number and viability. (I) IFN- $\gamma$ SFC by ELISPOT ( $P=0.0075$, one-way ANOVA; $n=3$ to 10). (J) Percent of $\mathrm{CD}^{+}$IFN- $\gamma^{+}$lymphocytes by flow cytometry $(P=0.0002$, one-way ANOVA; $n=3$ to 10$)$. $(\mathrm{K})$ Frequency of $\mathrm{CD} 4^{+}$lymphocytes producing cytokines $(P=0.0418$, one-way ANOVA; $n=4$ to 6). (L) LST induration size 48 hours after the injection of Leishmania antigen at 12 weeks after challenge $(P=0.0269$, oneway ANOVA; $n=3$ to 10). Cumulative data for $11 \mathrm{CTL}$ and 10 PdSP15 NHP from two independent experiments are shown. Lines and bars indicate the mean, and error bars indicate SEM.

Spearman correlation; $n=10$; fig. S4A). Because of the dichotomy of responses to PdSP15 immunization, we split PdSP15 vaccinated NHP to those that produced IFN- $\gamma\left(\mathrm{PdSP} 15-\mathrm{IFN}-\gamma^{+}\right)$or those where PdSP15 immunization induced a strong antibody and a poor IFN- $\gamma$ response (PdSP15-IFN- $\left.\gamma^{-}\right)$. Control (CTL)- and PdSP15-immunized NHP were challenged with 50 L. major-infected sand flies 1 month after the last immunization. Compared to controls, PdSP15-IFN- $\gamma^{+}$ (solid squares) NHP had significantly reduced disease burden $(P=0.0490$, one-

way ANOVA; $n=3$ to 11 ; Fig. $3 \mathrm{D}$ ) and reduced maximum lesion size $\left(P=0.0465\right.$, one-way ANOVA; $n=3$ to 11 ; Fig. 3E). PdSP15-IFN- $\gamma^{-}$ NHP (empty squares) were not protected and had a disease burden comparable to controls (Fig. 3, D and E). We did not observe a reduc-
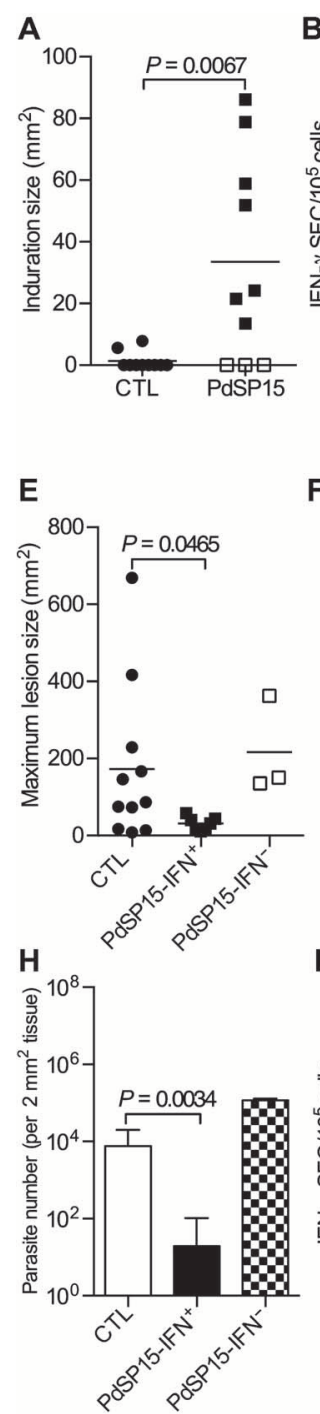
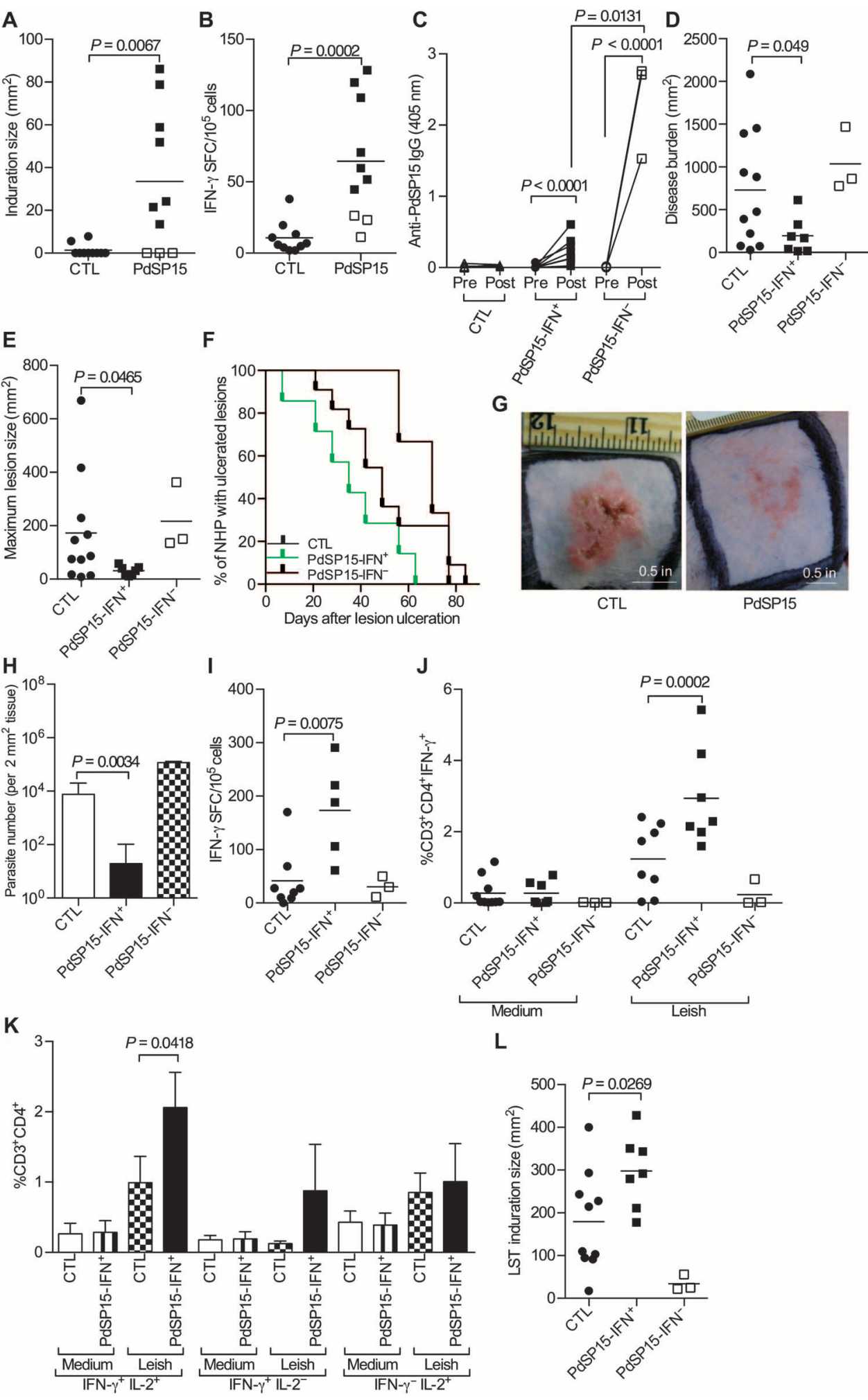

L

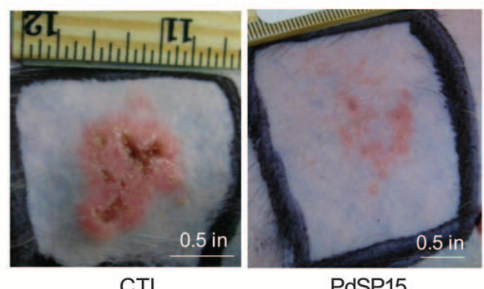

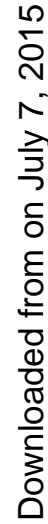

tion in the time to heal when comparing PdSP15-IFN- $\gamma^{+}$and controls (Fig. 3F). Representative photographs illustrate the reduction in lesion severity at 5 weeks after infection in PdSP15-IFN- $\gamma^{+}$NHP compared to controls (Fig. 3G). Disease amelioration was further echoed by a 
significant reduction in the number of parasites in PdSP15-IFN- $\gamma^{+}$ NHP compared to controls $(P=0.0034$, one-way ANOVA; $n=3$ to 8; Fig. $3 \mathrm{H})$. PdSP15-IFN- $\gamma^{-}$NHP harbored parasite numbers comparable to controls (Fig. $3 \mathrm{H}$ ).

To understand how cellular immunity to PdSP15 protects against vector-transmitted CL, we explored the early immune response to Leishmania in PdSP15-immunized NHP 2 weeks after challenge with infected sand flies. Similar to uninfected sand fly-exposed NHP, CD3 ${ }^{+}$ cells were the main source of specific anti-Leishmania IFN- $\gamma$ (fig. S4B). Compared to controls, PdSP15-IFN- $\gamma^{+}$NHP (solid squares) developed a stronger anti-Leishmania immune response after challenge, showing a significant increase in the number of IFN- $\gamma$ SFC $(P=0.0075$, one-way ANOVA; $n=3$ to 8 ; Fig. $3 \mathrm{I})$ and in the frequency of $\mathrm{CD} 4^{+} \mathrm{IFN}-\gamma^{+}$ lymphocytes ( $P=0.0002$, one-way ANOVA; $n=3$ to 10 ; Fig. 3 J). Notably, a significant increase in the proportion of Leishmania-specific $\mathrm{CD} 4^{+}$IFN $-\gamma^{+}$IL- $2^{+}$cells was also observed in PdSP15-IFN- $\gamma^{+}$NHP compared to controls ( $P=0.0418$, one-way ANOVA; $n=4$ to 6; Fig. $3 \mathrm{~K}$ ). Similar to uninfected sand fly-exposed NHP, both $\mathrm{CD}^{+}$and $\mathrm{CD} 4^{+}$ lymphocytes produced Leishmania-specific IFN- $\gamma$, but only the frequency of the latter was significantly higher in PdSP15-IFN- $\gamma^{+}$NHP compared to controls (Fig. 3J and fig. S4C), reinforcing the conclusion that the protective immune response is mostly driven by $\mathrm{CD}^{+}$lymphocytes. This rapidly developing robust immunity against Leishmania parasites was not observed in PdSP15-IFN- $\gamma^{-}$NHP (Fig. 3, I and J, empty squares). Our findings suggest that PdSP15-specific IFN- $\gamma$ promotes a microenvironment that facilitates priming of an early Leishmaniaspecific protective $\mathrm{CD} 4^{+} \mathrm{T}$ cell response.

In humans, the presence of a DTH after intradermal inoculation with killed Leishmania, known as a positive Leishmanin skin test (LST), is considered a signature of lifelong protective immunity against CL. Twelve weeks after infection, PdSP15-IFN- $\gamma^{+}$NHP had a significantly larger LST induration size (Fig. 3L, solid squares) compared to controls $(P=0.0269$, one-way ANOVA; $n=3$ to 11$)$ and to PdSP15-IFN- $\gamma^{-}$animals (Fig. 3L, empty squares). This suggests that infected controls and PdSP15-IFN- $\gamma^{-}$NHP developed a weaker immunity to Leishmania compared to PdSP15-IFN- $\gamma^{+}$NHP after resolution of the infection.

\section{PdSP15 is a member of the insect family of odorant-binding proteins with no sequence or structure homology to known human proteins}

The protective salivary antigen PdSP15 shares sequence homology only to the small odorant-binding protein family found exclusively in the salivary glands of sand flies (Fig. 4A), with 67 and 54\% identity to the P. papatasi and Phlebotomus sergenti salivary proteins PpSP15 and PsSP15, respectively (Fig. 4B). To exclude any structural similarities to human proteins, the crystal structure of PdPS15 was solved to a $2.95-\mathrm{nm}$ resolution (Fig. $4 \mathrm{C}$, table S3). The structure is available at the Research Collaboratory for Structural Bioinformatics Protein Data Bank (RCSB PDB) with PDB code 4OZD. PdSP15 contains six $\alpha$-helical elements designated a, $c, d, e, f$, and $g$ that match the homologous secondary structures of insect odorant-binding proteins. Helix e is elongated relative to other described insect proteins and contains a number of basic (arginine and lysine) residues. Structural search with the program DALI (12) showed a distant similarity to insect odorant-binding protein family members including the D7 proteins found in the saliva of mosquitoes and did not identify structural similarities to mammalian proteins (fig. S5).

\section{PdSP15 is immunogenic in humans naturally exposed to $P$. duboscqi bites}

Having established that PdSP15 is an antigen foreign to humans, we investigated the immunogenicity of rPdSP15 in individuals naturally exposed to P. duboscqi bites (13). Sand fly-exposed individuals with antibodies to whole saliva produced significant levels of antibodies to $\mathrm{rPdSP} 15$ ( $P<0.0001$, Mann-Whitney test; $n=12$ to 30; Fig. 5A) or the SGH $(P<0.0001$, Mann-Whitney test; $n=12$ to 30 ; Fig. $5 \mathrm{~A})$. PBMCs from 14 individuals naturally exposed to $P$. duboscqi bites (18 to 65 years old) were stimulated with SGH or rPdSP15 in vitro, and supernatants were collected 96 hours after stimulation. Levels of IFN- $\gamma$, IL-10, IL-17, IL-5, and IL-13 were detected by a Luminex assay (Fig. 5B). Levels of IL-2, IL-4, and IL-9 in these samples were below the limit of detection of the assay. Compared to controls, stimulation with SGH induced significant levels of IFN- $\gamma$ (mean, $294.6 \mathrm{pg} / \mathrm{ml} ; P=0.0354$, one-way ANOVA; $n=14$; Fig. 5B), IL-10 (mean, $32.47 \mathrm{pg} / \mathrm{ml} ; P=0.0112$, oneway ANOVA; $n=14$; Fig. 5B), IL-17 (mean, 245.4 pg/ml; $P=0.0004$, oneway ANOVA; $n=14$; Fig. 5B), and IL-5 (mean, $65.27 \mathrm{pg} / \mathrm{ml} ; P=0.0344$,

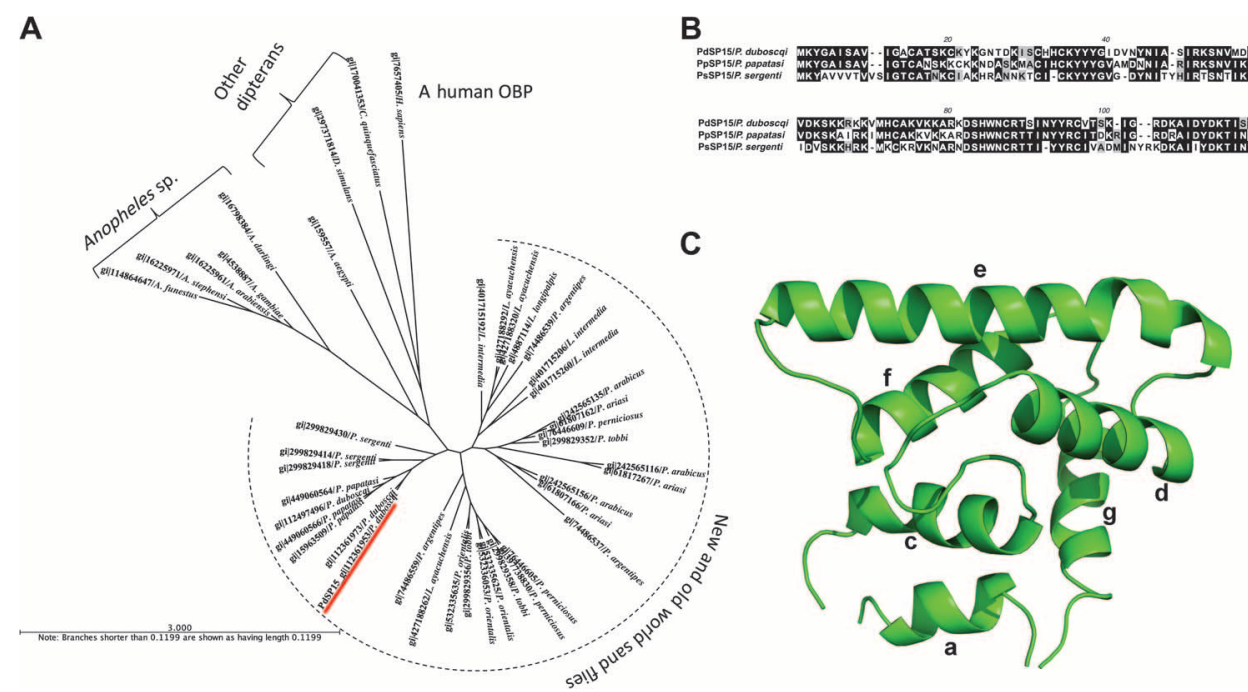

Fig. 4. PdSP15 is an odorant-binding protein in saliva of phlebotomine sand flies. (A) Phylogenetic tree analysis shows the similarity of odorant-binding proteins in New and Old World sand fly species and their divergence from odorant-binding proteins (OBP) of other dipterans and humans. Bootstrap value, 10,000. PdSP15 location is underlined in red. (B) Sequence alignment between PdSP15 from P. duboscqi (accession number 112361953) and its orthologs in P. papatasi (PpSP15, accession number 449060564) and $P$. sergenti (PsSP15, accession number 299829414). Black shading and gray shading represent identical and similar amino acids, respectively. (C) Crystal structure of PdSP15 (4OZD) containing six $\alpha$-helical elements designated as $a, c, d, e, f$, and $g$. 
A
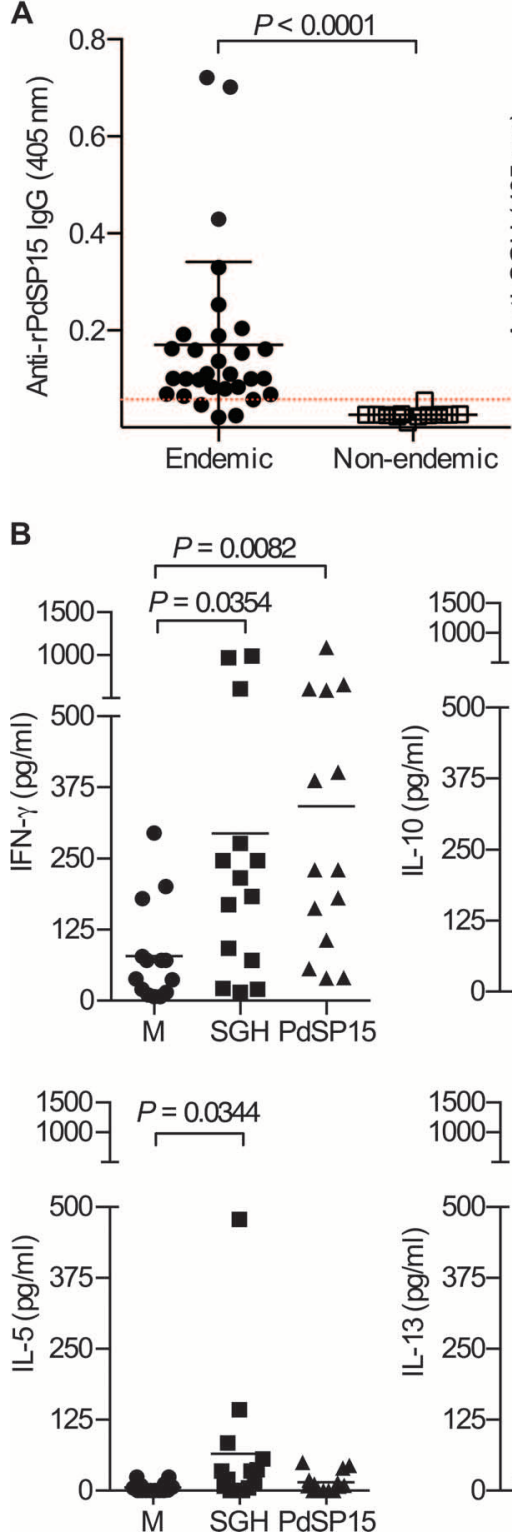
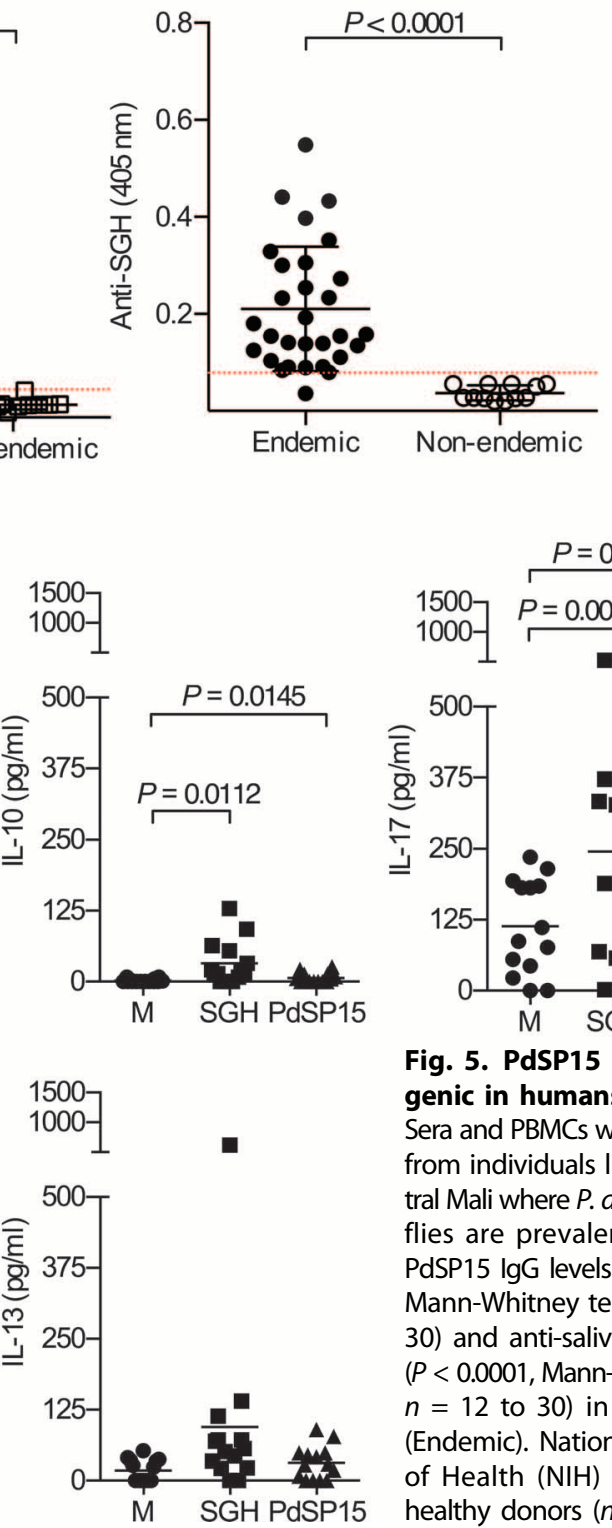
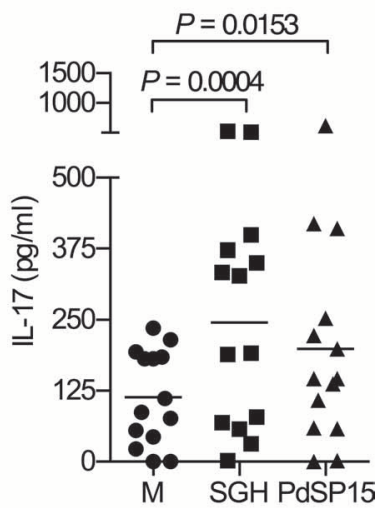

Fig. 5. PdSP15 is immunogenic in humans. (A and $\mathbf{B}$ ) Sera and PBMCs were obtained from individuals living in central Mali where $P$. duboscai sand flies are prevalent. (A) AntiPdSP15 lgG levels $(P<0.0001$, Mann-Whitney test; $n=12$ to 30) and anti-saliva (Anti-SGH) $(P<0.0001$, Mann-Whitney test; $n=12$ to 30 ) in 30 Malians (Endemic). National Institutes of Health (NIH) blood bank healthy donors $(n=12)$ were used as controls (Non-endemic).

Dashed lines indicate the cutoff (calculated as the mean in the non-exposed group \pm 2.5 SD). (B) Cytokine levels produced by PBMCs from 14 Malian villagers after stimulation with SGH or PdSP15: IFN- $\gamma$ (mean, $294.6 \mathrm{pg} / \mathrm{ml} ; P=0.0354$, one-way ANOVA; $n=14$ ), IL-10 (mean, $32.47 \mathrm{pg} / \mathrm{ml}$; $P=0.0112$, one-way ANOVA; $n=14$ ), IL-17 (mean, $245.4 \mathrm{pg} / \mathrm{ml} ; P=0.0004$, one-way ANOVA; $n=14$ ), and IL-5 (mean, $65.27 \mathrm{pg} / \mathrm{ml} ; P=0.0344$, one-way ANOVA; $n=14$ ), or stimulation with $\mathrm{rPdSP} 15$ yielded IFN- $\gamma$ (mean, $342 \mathrm{pg} / \mathrm{ml} ; P=0.0082$, one-way ANOVA; $n=14$ ), IL-10 (mean, $6.757 \mathrm{pg} / \mathrm{ml}$; $P=0.0145$, one-way ANOVA; $n=14$ ), and IL-17 (mean, $198.7 \mathrm{pg} / \mathrm{ml} ; P=0.0153$, one-way ANOVA; $n=14$ ), but not IL-5 (mean, $14.68 \mathrm{pg} / \mathrm{ml} ; P=0.7409$, one-way ANOVA; $n=14$ ). PBMCs were selected on the basis of cell viability. Lines indicate the mean.

one-way ANOVA; $n=14$; Fig. 5B), whereas stimulation with rPdSP15 induced significant levels of IFN- $\gamma$ (mean, $342 \mathrm{pg} / \mathrm{ml} ; P=0.0082$, one-way ANOVA; $n=14$ ), IL-10 (mean, $6.757 \mathrm{pg} / \mathrm{ml} ; P=0.0145$, one-way ANOVA; $n=14$ ), and IL-17 (mean, $198.7 \mathrm{pg} / \mathrm{ml} ; P=0.0153$, oneway ANOVA; $n=14$ ), but not IL-5 (mean, $14.68 \mathrm{pg} / \mathrm{ml}$ ). Production of IL-13 was not different from control (M) upon stimulation with SGH or PdSP15 (Fig. 5B).

\section{DISCUSSION}

To date, there are no licensed human vaccines against any form of human leishmaniasis. Here, we adopt an innovative approach where we use an immunogenic protein from saliva of $P$. duboscqi, a natural vector of $L$. major, as a preventive vaccine against CL. We show that compared to controls, NHP exposed to uninfected sand fly bites or immunized with a defined recombinant salivary protein, rPdSP15, controlled sand fly-transmitted L. major infection, showing a significant reduction in disease burden, maximum lesion size, and parasite numbers in lesions. Additionally, protected NHP developed an early and more robust Leishmaniaspecific immune response, observed in studies using rodents $(2,14)$. This study demonstrates the protective efficacy of a salivary antigen from a sand fly vector against leishmaniasis in NHP, reinforcing previous findings in rodent models of infection $(15,16)$. In combination with parasite antigen/s, targeting vector-derived molecules as a component of Leishmania vaccines presents an opportunity to improve their effectiveness. In a recent study, using various vaccine modalities of PpSP15 DNA, a $\mathrm{T}_{\mathrm{H}}$ 1-DTH-inducing salivary protein from $P$. papatasi that protected mice from L. major infection as a DNA vaccine $(3,6)$, and live recombinant Leishmania tarentolae stably expressing cysteine proteinase genes (17), the strongest protective effect was observed when priming with PpSP15 DNA and boosting with PpSP15 DNA plus the recombinant parasite (18), suggesting that priming with a sand fly salivary protein and boosting with the salivary protein in the presence of a Leishmania antigen may improve protective immunity.

The primary mechanism of protection from CL through sand fly saliva-mediated immunity may be the outcome of indirect killing of a significant number of parasites by saliva-specific $\mathrm{T}_{\mathrm{H}} 1$ cells within the DTH site that also generates an early Leishmania-specific immune response. Alternatively, the immune response to uninfected sand fly bites may generate an adjuvanting effect in the skin, priming a $\mathrm{T}_{\mathrm{H}} 1$ anti-parasite immune response that is instrumental to the observed protection. Possibly, it is a combination of the two. Independent of the significance of the observed Leishmania-specific immune response to the primary challenge, it would certainly ensure long-term protection against subsequent infections with $L$. major. It is worth noting that the NHP used in this study were challenged with $L$. major-infected P. duboscqi bites. Compared to needle-injected parasites, sand flyinitiated CL has been shown to result in a more virulent infection in rodent models $(7,8,19)$. Vaccine candidates that protect rodents against a challenge with needle-injected parasites may fail against an infected sand fly challenge $(8,20)$. This indicates that anti-Leishmania vaccine 
candidates that protect against a stringent vector challenge are likely to fare better in clinical settings.

Of significance, PdSP15 from P. duboscqi is a homolog of PpSP15 from P. papatasi and PsSP15 from P. sergenti. The high homology of PdSP15, PpSP15, and PsSP15 suggests that PdSP15 may also protect against disease transmitted by the most significant Leishmaniainfected Old World vectors of CL. Substantiating evidence is demonstrated by the cross-protection of $P$. papatasi-exposed mice against P. duboscqi salivary gland extract plus L. major (21). Notably, $90 \%$ of 200 children from a CL endemic area in Tunisia, where L. major is transmitted by $P$. papatasi, also recognized a protein of $15 \mathrm{kD}$, likely PpSP15, by Western blot $(22,23)$. Also important to vaccine development, PdSP15 is foreign to humans. The structure of PdSP15 belongs to the family of insect odorant-binding proteins. The odorant-binding proteins in mammals have an unrelated fold consisting of an antiparallel $\beta$ barrel that places them in the lipocalin protein family (24), whereas insect odorant-binding proteins are all $\alpha$-helical and have no mammalian homologs (25).

PdSP15 was identified using a limited number of NHP by the RAS approach $(14,26)$. RAS also offers other advantages over traditional screening strategies, enabling the simultaneous testing of various immunogens in a single animal, eliminating the high costs and hurdles of producing endotoxin-free recombinant proteins through the use of DNA plasmids, and insuring that the vaccine candidate elicits an immune response formed against the native protein. Hence, this approach could be adapted as a translational tool for other infectious diseases and consequently accelerate the identification of vaccine candidates for human clinical trials.

The prevention of leishmaniasis through vaccination has remained an elusive target to date despite the multitude of Leishmania-based antigens and adjuvants that have been evaluated as vaccines (27-29). Salivary proteins may promote Leishmania vaccine efficacy by generating an immune response that targets the site of bite. Considering that infection with any form of leishmaniasis is initiated in the skin, inclusion of a salivary antigen in a Leishmania vaccine would potentiate the host immune response at this early stage of the infection, targeting parasites when they are lowest in number. Additionally, they may offer long-term memory through natural boosting by uninfected bites, a major obstacle to development of an effective Leishmania vaccine.

Despite the above-mentioned promising findings, this study has its limitations. Vector transmission results in a higher inoculum variability compared to needle injection, because infected flies egest a variable number of parasites (30-32) and their feeding/probing behavior is uneven. This was overcome by using 50 Leishmania-infected $P$. duboscq $i$ sand flies that resulted in a $90 \%$ successful infection rate, an improvement over previous reports of infection rates in NHP after vector transmission of L. major (33). The use of 50 infected flies resulted in clusters of lesions that resembled human CL evolving from nodules to papules to ulcerated lesions with elevated borders and a necrotic center before self-healing over the course of 14 weeks. Another limitation to this study is that only 63 and $70 \%$ of NHP exposed to noninfected bites or immunized with PdSP15, respectively, developed a cell-mediated immune response to the salivary components. We credit the outbred nature of the NHP as a possible factor in the observed differences in their immune response to saliva or PdSP15. Indeed, only 50\% of human subjects experimentally exposed to bites of Lutzomyia longipalpis sand flies presented with nodules indicative of a DTH response (34). Moreover, 23 and $25 \%$ of PBMCs from individuals inhabiting an endemic area of CL in central Mali where P. duboscqi is the main vector of L. major (35) displayed a $\mathrm{T}_{\mathrm{H}} 1$ - or $\mathrm{T}_{\mathrm{H}} 2$-polarized response upon stimulation with whole saliva of $P$. duboscqi, and $52 \%$ presented with a mixed response (13). The factors underlying the absence or diversity of the cellular immune response against sand fly salivary proteins in some humans and NHP remain to be elucidated and may be of importance in determining who in endemic areas are likely to contract CL. Possibly, the absence of immunity in a subset of humans could be overcome with different vaccination strategies, using adjuvants or adenovirus-based DNA vaccines.

A positive outcome to the observed dichotomy of the immune response to PdSP15 is the verification that a $\mathrm{T}_{\mathrm{H}} 1$ cell-mediated immunity, unlike a humoral immune response, to $\mathrm{PdSP} 15$ correlates to protection from CL. Compared to controls and PdSP15-IFN- $\gamma^{-}$NHP, PdSP15-IFN- $\gamma^{+}$ NHP controlled the sand fly-transmitted L. major infection, showing a reduction in disease burden, maximum lesion size, and parasite numbers, as well as an accelerated protective Leishmania-specific immune response. Protected PdSP15-IFN- $\gamma^{+}$NHP also developed a significantly higher frequency of $\mathrm{CD}^{+} \mathrm{CD} 4^{+} \mathrm{IFN}-\gamma^{+}$-producing cells when stimulated with Leishmania antigen compared to controls and unprotected PdSP15-IFN- $\gamma^{-}$NHP. Moreover, we observed a higher frequency of polyfunctional cells producing IFN- $\gamma$ and IL-2 cytokines in PdSP15-IFN- $\gamma^{+}$ NHP, although tumor necrosis factor- $\alpha$ (TNF- $\alpha$ ) was undetectable. The presence of polyfunctional $\mathrm{T}$ cells has been associated with effectiveness of the protective immune response in several vaccine candidates for diverse infectious diseases such as leishmaniasis (36), smallpox (37), hepatitis C virus (38), and tuberculosis (TB) $(39,40)$. Higher frequencies of these cells were also present in HIV nonprogressors (41). Hence, induction of polyfunctional $\mathrm{T}$ cells can be taken as an indication of the superior quality of the immune response (42). The robustness of the immunity against Leishmania in PdSP15-IFN- $\gamma^{+} \mathrm{NHP}$ is further supported by their development of larger LST measurements compared to controls and PdSP15-IFN- $\gamma^{-}$NHP. LST has been widely used as a diagnostic tool for CL in humans, indicating exposure to, and immunity against, Leishmania parasites (43-45) and a good indicator of vaccine efficacy in Leishmania-based vaccine candidates (46-48). Notably, in an endemic area of CL in Tunisia, the size of the LST reaction positively correlated to resistance to reinfection (49). The comparable level of protection observed in PdSP15-IFN- $\gamma^{+}$NHP and animals exposed to uninfected sand fly bites suggests that PdSP15, one of the most abundant salivary protein in $P$. duboscqi, is the main driver of the protective immune response observed after challenge of NHP exposed to uninfected sand fly bites.

The use of a salivary molecule in a Leishmania vaccine poses another challenge. Inhabitants of leishmaniasis endemic regions are well exposed to salivary proteins of vector sand flies through the multitude of uninfected bites they receive daily. Moreover, vaccinated naïve individuals are likely to be bitten frequently by the vector during their stay in the endemic area. Our findings suggest that PdSP15 represents a promising vaccine candidate for saliva-naïve individuals such as tourists and military personnel. However, whether vaccination with PdSP15 would recall or drive a predominant $\mathrm{T}_{\mathrm{H}} 1$ response in endemic populations remains to be tested. Here, NHP exposed to uninfected bites and inoculated with PdSP15 DNA recalled such a response, arguing for its potential usefulness also in CL endemic areas. We should also emphasize that none of the immunized NHP developed an adverse reaction to $\mathrm{rPdSP} 15$. Nevertheless, these issues need to be addressed in the context of possible allergic reactions and long-term safety. 
It is worth noting that $\mathrm{rPdSP} 15$ was expressed in Escherichia coli, refolded, solubilized, and purified without difficulty while retaining its immunogenicity. Therefore, it promises to be amenable for commercial scale production required of viable vaccine candidates. To establish the translational relevance of the study findings, we tested the immunogenicity of rPdSP15 in inhabitants of an endemic area of CL in central Mali where P. duboscqi is the main vector of $L$. major (35). We show that 93 and $87 \%$ of 30 individuals produce antibodies to whole saliva or rPdSp15, respectively. Moreover, stimulation of PBMCs from 14 individuals with SGH or rPdSP15 induced significant levels of IFN- $\gamma$, IL-10, and IL-17 compared to medium. When compared to stimulation with SGH, rPdSP15 stimulation induced comparable levels of IFN- $\gamma$ (116.1\%), but only 20.1 and $22.5 \%$ of IL-10 and IL-5 levels, respectively, inferring the specificity of $\mathrm{rPdSP} 15$ as a $\mathrm{T}_{\mathrm{H}} 1$-inducing salivary protein in humans. As for IL-17, it has been associated to tissue damage in chronic phases of mucosal leishmaniasis and CL lesions (50); however, its role in leishmaniasis remains under scrutiny (51). Less is known about IL-17 and sand fly saliva (52). In mice, a DTH response is inhibited if IL-17 is absent (53). The established association between the development of a $\mathrm{T}_{\mathrm{H}} 1-\mathrm{DTH}$ response and salivamediated protection against leishmaniasis suggests that $\mathrm{IL}-17$ production may be of importance in anti-saliva immunity. Moreover, IFN- $\gamma$ and IL-17 can be synergistically produced by activated CD4 ${ }^{+} \mathrm{T}$ cells in the skin and induce keratinocytes to produce a proinflammatory environment (54) that would be detrimental to the establishment of the parasite. Notably, the observed proinflammatory response to rPdSP15 in humans naturally exposed to bites of $P$. duboscqi reinforces the likelihood that inhabitants of leishmaniasis endemic areas that are constantly exposed to vector bites would also benefit from a vaccine containing an appropriate salivary antigen.

Here, we provide solid preclinical data in NHP regarding the efficacy of rPdSP15 as a preventative vaccine against vector-transmitted L. major infection. We also demonstrate its ability to recall a proinflammatory response in saliva-exposed humans, advancing its potential as a human vaccine for CL. Future studies will focus on the scalability and safety of rPdSP15 in preparation for a first-in-humans clinical trial.

\section{MATERIALS AND METHODS}

\section{Study design}

This study was designed to investigate the efficacy of $P$. duboscqi sand fly salivary proteins as vaccine candidates against CL. We exposed rhesus macaques to uninfected sand fly bites or immunized them with an immunogenic sand fly salivary protein (PdSP15). Next, we challenged exposed or immunized groups of animals via L. major-infected sand fly bites. We followed the clinical evolution of the CL lesions by size and parasite number. Our study endpoint was CL cure, defined as the complete cicatrization of skin ulcers. We used ELISPOT and flow cytometry to analyze immunological parameters specific to the sand fly PdSP15 salivary protein or to parasite crude antigen (Leish). For challenge experiments, we calculated our sample sizes to achieve statistically significant results if protection is at least $80 \%$ effective (a $0.835 \%$ probability of preventing or reducing the CL outcome) when considering that $90 \%$ of the naïve NHP would develop lesions after challenge. Data from available frozen sera and cryopreserved PBMCs from inhabitants of an endemic area where P. duboscq $i$ is prevalent were used to assess the immunogenicity of PdSP15 in humans. We analyzed the human antibody response and cytokine production in response to PdSP15 recombinant salivary protein. Investigators were blinded when conducting all immunizations (use of coded vials for PdSP15 and control immunizations) and throughout the course of the experimental CL disease (NHP ID and group allocation were coded). The number of animals per group and per experiment is indicated in all figure legends. NHP were randomized by sex, age, and weight. Research was conducted in compliance with the Animal Welfare Act, ARRIVE guidelines, and other federal statutes and regulations relating to animals and experiments involving animals and adheres to principles stated in the Guide for the Care and Use of Laboratory Animals, MRC Publication, 2011 edition.

\section{Animals}

Rhesus macaques (Macaca mulatta, Indian strain) were housed in the Walter Reed Army Institute of Research vivarium where manipulations were conducted under protocol IEO02-09 approved by the Walter Reed Army Institute of Research Institute Animal Care and Use Committee and by the National Institute of Allergy and Infectious Diseases (NIAID) Animal Care and Use Committee under protocol LMVR12. Because of reports of enhanced resistance to CL in rhesus monkeys of Chinese origin, we used NHP of Indian origin that have been shown to be susceptible to L. major parasites (33). All NHP were screened for good physical health and absence of antibodies reactive to $P$. duboscq $i$ sand fly salivary proteins before enrollment into the animal protocol. During experimental manipulations, they were housed individually in stainless steel cages (cubic $\sim 2 \mathrm{~m}$ ), kept in environmentally controlled rooms with 10 to 15 air changes per hour, temperature range $18^{\circ}$ to $29^{\circ} \mathrm{C}$, relative humidity $70 \%$, and light/dark 12:12-hour cycle. NHP were fed a staple diet of LabDiet Primate Chow \#5038, supplemented by Prima Treats \#F05709 (Bio-Serve) fresh fruits and vegetables with water available ad libitum from an automatic system. Macaques were consistently negative for SIV (simian immunodeficiency virus) and STLV (simian $\mathrm{T}$ lymphotrophic virus) when serologically tested annually, and quarterly, intradermal TB tests were negative. During the course of these experiments, 75 M. mulatta were tested, comprising both sexes, aged 4 to 12 years and weighing 3.5 to $12.7 \mathrm{~kg}$. Procedures for exposing NHP to sand flies generally followed those of Probst et al. (55). The Walter Reed Army Institute of Research Animal Use Program is fully accredited by the Association for Assessment and Accreditation of Laboratory Animal Care (AAALAC).

\section{Sand flies and SGH}

P. duboscqi sand flies originally from Mali, West Africa, and reared in the insectary facilities of the Laboratory of Malaria and Vector Research, NIAID, NIH, were used for the described experiments. For transmission experiments, 3- to 4-day-old sand flies were allowed to feed on blood containing L. major promastigotes as previously described (4). Sand flies with mature infections (11 to 15 days after blood feeding) were used to transmit Leishmania parasites to NHP. Five- to 7-day-old sand flies were used for preparation of SGH. Briefly, pools of 20 salivary glands were dissected in phosphate-buffered saline. SGH was prepared by ultrasonication followed by centrifugation at $10,000 \mathrm{~g}$ for $3 \mathrm{~min}$ at $4{ }^{\circ} \mathrm{C}$.

\section{Parasites}

L. major WR 2885 strain was used to infect sand flies and for preparation of specific Leishmania antigen. This strain of parasites was recently isolated from a soldier deployed to Iraq as previously described (4). 
L. major were grown at $26^{\circ} \mathrm{C}$ in Schneider's medium supplemented with $10 \%$ heat-inactivated fetal calf serum (Life Technologies), penicillin $(100 \mathrm{U} / \mathrm{ml})$, streptomycin $(100 \mu \mathrm{g} / \mathrm{ml}), 2 \mathrm{mM}$ L-glutamine. Leishmania antigen was prepared by harvesting $1 \times 10^{9}$ parasites from culture flasks and repeated freeze-thaw cycles. Protein amounts in the Leishmania homogenate were determined by bicinchoninic acid (BCA) protein quantification. Aliquots $(1 \mathrm{mg} / \mathrm{ml})$ were frozen at $-7^{\circ} \mathrm{C}$ until use.

\section{Production of DNA plasmids coding for $P$. duboscqi salivary proteins}

Twenty-three DNA plasmids coding to $P$. duboscqi salivary proteins were cloned in the VR2001-TOPO vector (Vical Inc.), and endotoxinfree DNA was purified as previously described (14). The transcripts cloned were PduM01 (DQ826517), PdSP15 (DQ826514), PduM03 (DQ826524), PduM04 (DQ834339), PduM06 (DQ826516), PduM07 (DQ826518), PduM10 (DQ826519), PduM12 (DQ826521), PduM29 (DQ826515), PduM31 (DQ826520), PduM33 (DQ834330), PduM34 (DQ8356), PduM35 (DQ826522), PduM39 (DQ834331), PduM46 (DQ826523), PduM48 (DQ826525), PduM49 (DQ826526), PduM51 (DQ835384), PduM54 (DQ834338), PduM57 (DQ834333), PduM72 (DQ835356), PduM99 (DQ835359), and PduM120; these DNA plasmids were diluted in normal saline (Baxter), filtered through a $0.2-\mu \mathrm{m}$ pore (Millipore), and kept frozen until use.

\section{Reverse antigen screening}

NHP were inoculated intradermally with $30 \mu \mathrm{g}$ of the 23 distinct DNA plasmids, once, for reverse antigen experiments. Briefly, NHP were exposed to uninfected sand fly bites three times, every 21 days. One month after the last uninfected sand fly bite exposure, NHP were injected intradermally using an insulin syringe with each of the DNA candidates, empty plasmid control, SGH, and bites from one uninfected sand fly in the inner thighs and/or chest. Inoculations were placed far from each other, and each placement was marked with permanent ink in the NHP skin and recorded in transparency films. Twenty-four and 48 hours after the inoculations, reactions were recorded by measurement of the induration diameter suing a Vernier caliper. Positive reactions and empty plasmid controls were biopsied with a $2-\mathrm{mm}$ punch at 48 hours after the injections. Tissue samples were preserved in RNA later until use.

\section{Production of recombinant protein PdSP15}

The complementary DNA (cDNA) for PdSP15 (accession number DQ826514) was modified using PCR to remove the signal sequence and insert an initiator methionine codon at the $5^{\prime}$ end. This fragment was cloned into the expression vector pET17b, and the vector was moved into the E. coli strain BL21(DE3)pLysS for expression. Inclusion bodies produced by previously described methods were dissolved in $6 \mathrm{M}$ guanidine $\mathrm{HCl}$ ( $\mathrm{pH} \mathrm{8.0)}$ ), containing $10 \mathrm{mM}$ dithiothreitol, and refolded by dilution into a large volume of $20 \mathrm{mM}$ tris- $\mathrm{HCl}(\mathrm{pH} 8.5)$, $300 \mathrm{mM}$ arginine $\mathrm{HCl}$. The refolded protein was concentrated by ultrafiltration and purified by a combination of gel filtration (Sephacryl S-100) and cation exchange (SP Sepharose) chromatography. Any remaining lipopolysaccharide was removed using ToxinEraser Endotoxin Removal Kit (GenScript) following the manufacturer's protocol.

\section{Crystallization and structure determination of PdSP15}

Recombinant PdSP15 was crystallized using the hanging drop vapor diffusion method with a precipitant containing 10\% PEG 6000 (polyethylene glycol, molecular weight 600), 0.1 M MES (pH 6.0). Crystals were flash- frozen for data collection after transfer to the crystallization condition supplemented with $20 \%$ glycerol. Diffraction data collection was performed at beamline 19-ID at the Structural Biology Center, Advanced Photon Source (APS), Argonne National Laboratory. PdSP15 crystallized in the space group $\mathrm{P} 2{ }_{1} 2_{1} 2_{1}$ with seven molecules in the asymmetric unit, and the crystals diffracted to a resolution of $2.95 \AA$. The structure of PdSP15 was determined by molecular replacement using a monomeric PdSP15b structure (56) as a search model in the program PHASER (57). Protein sequence alignment was performed on the MacVector software following Dayhoff matrix for identical and similarity shading format.

\section{Immunization of NHP with salivary PdSP15}

NHP were inoculated intradermally in the inner thigh with $500 \mu \mathrm{g}$ of DNA plasmid coding to PdSP15, twice, 21 days apart followed by an intradermal protein boost in the opposite inner thigh with $30 \mu \mathrm{g}$ of rPdSP15 with $20 \mu \mathrm{g}$ of GLA-SE. Recombinant PdSP15 was mixed in solution with GLA-SE right before inoculations.

\section{Challenge of immunized NHP with L. major-infected sand flies}

One month after the PdSP15 protein boost, PdSP15- and controlimmunized animals were exposed in the chest to $50 \mathrm{~L}$. major-infected sand flies kept in a Plexiglas feeding device with one mesh surface that allows the sand flies to probe and feed in the NHP skin. The feeding device was left on the NHP chest for $25 \mathrm{~min}$. Lesion size was measured weekly as the diameter of the skin lesion using a digital Vernier caliper (Mitutoyo). Disease burden was calculated as the cumulative area of lesions over the time; maximum lesion diameter was determined as the largest lesion measurement at any time during the disease course. The time to heal is the cumulative measurement in days of lesion development starting at the time of ulceration, which varies between animals, until complete scarring is observed. All lesions and bite reactions were photographed.

\section{Leishmanin skin test}

Animals were inoculated with $100 \mu \mathrm{g}$ in $100 \mu \mathrm{l}$ of the Leishmania antigen, intradermally in the left inner thigh. Forty-eight hours later, skin reaction was read using a ballpoint pen to determine the size of the induration (58). Measurements were taken with a Vernier caliper (Mitutoyo).

\section{Antibody measurements by enzyme-linked immunosorbent assay}

Microtiter plates were coated with $P$. duboscqi sand fly SGH (1 pair/ $\mathrm{ml})$ or with $\mathrm{rPdSP} 15(2 \mu \mathrm{g} / \mathrm{ml})$ overnight at $4{ }^{\circ} \mathrm{C}$. Plates were blocked with tris-buffered saline, $4 \%$ bovine serum albumin, $0.05 \%$ Tween 20 (Sigma) for 2 hours. Sera were diluted (1:100) and incubated for 2 hours at $37^{\circ} \mathrm{C}$. After washes, goat anti-monkey IgG (Bethyl) or goat anti-human IgG (Sigma) was diluted $(1: 10,000)$ and incubated for 1 hour. Alkaline phosphatase substrate (Sigma) was added for $30 \mathrm{~min}$, and absorbance was read at $405 \mathrm{~nm}$ in a spectrophotometer (Molecular Devices).

\section{Histological analysis of DTH site}

Two-millimeter punch biopsy was taken from the NHP skin at the site of sand fly bite. Tissue was fixed in 10\% buffered formalin and preserved in paraffin blocks. Sections were stained with hematoxylin and eosin (Histoserv).

\section{Cytokines and parasite quantification by qPCR}

A 2-mm punch skin biopsy was taken from the site of the sand fly bite or from a lesion border and preserved in Allprotect solution (Qiagen) 
until use. DNA or RNA was extracted using QIAamp DNA Micro Kit or RNeasy Mini Kit, respectively, following the manufacturer's instructions. From total RNA (100 ng), cDNA was synthesized using qScript cDNA SuperMix (Quanta BioSciences). Relative quantification of cytokines was achieved using the Universal ProbeLibrary system (Roche). Probes \#20 for IL-4 and \#21 for IFN- $\gamma$ in conjunction with specific primers (IFN- $\gamma$ : $5^{\prime}$-tggaaagaggagagtgacagaa- $3^{\prime}$ and $5^{\prime}$-tggatcctctggtcatctttg- $3^{\prime}$; IL-4: $5^{\prime}$-gaaacggctcgacaggaac- $3^{\prime}$ and $5^{\prime}$-andtttccaagaagtcttccaacg- $3^{\prime}$ ) were determined by the ProbeLibrary software version 2.5 (Roche) to be specific for these cytokines. Numbers of parasites in the skin were determined by SYBR Green real-time PCR assay and primers JW11-JW12 as targets for Leishmania amplification (59). Relative quantification of target genes versus $18 S$ reference gene was calculated on the LightCycler 480 software (Roche). Normal skin samples were used as calibrators, and relative expression was calculated by the $\Delta \Delta C_{\mathrm{T}}$ method and then divided by the value obtained for the calibrators. Results are expressed as relative expression, where value of 1 is the normal skin baseline.

\section{Blood withdrawal and cell culture}

Blood was collected from the femoral vein of anesthetized animals into heparin-coated Vacutainer tubes for PBMC separation and BD Vacutainer SST serum separation tubes. Sera were collected after a brief centrifugation and stored at $-20^{\circ} \mathrm{C}$ until use, following the manufacturer's instructions. PBMCs were separated by gradient centrifugation and cryopreserved in a solution of $10 \%$ dimethyl sulfoxide, $50 \%$ fetal bovine serum, and $40 \%$ RPMI. Cells were thawed at $37^{\circ} \mathrm{C}$ water bath, washed twice, and seeded at $1 \times 10^{6} / \mathrm{ml}$ or $2 \times 10^{5} / \mathrm{ml}$ for flow cytometry or ELISPOT assays, respectively. Cells were stimulated with Leishmania antigen $(50 \mu \mathrm{g} / \mathrm{ml})$ or $\mathrm{rPdSP} 15(10 \mu \mathrm{g} / \mathrm{ml})$ or anti-CD3 $(100 \mathrm{ng} / \mathrm{ml})$ (Mabtech) in combination with CD28 $(2 \mathrm{mg} / \mathrm{ml})$ and CD49d $(2 \mathrm{mg} / \mathrm{ml})$ (BioLegend).

\section{Immune correlates of protection by flow cytometry and ELISPOT}

Staining of PBMCs was undertaken after 2 hours of stimulation with antigens followed by brefeldin A (1:1000) for the last 16 hours of incubation. Cells were harvested and stained with LIVE/DEAD Fixable Yellow Stain Kit (Life Technologies). Cells were blocked with 10\% fetal bovine serum and surface-stained with anti-CD3 (BD Biosciences, SP34-2), anti-CD4 (BD Biosciences, L200), and anti-CD8 (BD Biosciences, RPA-T8). Intracellular staining was achieved by Cytofix/ Cytoperm kit (BD Biosciences) following the manufacturer's instructions. Cells were stained with anti-IFN- $\gamma$ (BioLegend, B27), anti-IL-2 (BioLegend, MQ1-17H2), and anti-TNF- $\alpha$ (BD Biosciences, MAB11). Data were acquired in a MACSQuant Analyzer and analyzed using FlowJo version 9.5.6 software (Tree Star). Gates indicating the production of intracellular cytokines were set on the basis of negative controls (fig. S2A). Cells were plated and stimulated for 48 hours with distinct antigens during the IFN- $\gamma$ ELISPOT assay. ELISPOT was performed following the manufacturer's recommendations (Mabtech). Image capture and analysis of the number of spots were performed by a CTL-ImmunoSpot Analyzer and software (Cellular Technologies).

\section{Human cell culture and cytokine measurement}

Frozen human PBMCs from 14 healthy permanent residents (age 18 to 65 years) of a CL endemic area of central Mali were thawed and resuspended in RPMI 1640 medium supplemented with 10\% AB human serum,
$1 \%$ sodium pyruvate, $1 \%$ nonessential amino acids, $1 \%$ Hepes buffer, $0.05 \%$ $\beta$-mercaptoethanol (1000 $\times)$, and penicillin/streptomycin $(2 \mu \mathrm{g} / \mathrm{ml})$. Cells were cultured in 96-well plates at $1 \times 10^{6} \mathrm{cells} / \mathrm{ml}$ in a final volume of $200 \mu \mathrm{l}$ and incubated with SGE (1 pair/ml), rPdSP15 $(5 \mu \mathrm{g} / \mathrm{ml})$, or ConA $(2.5 \mu \mathrm{g} / \mathrm{ml})$ in a $5 \% \mathrm{CO}_{2}$ humidified atmosphere at $37^{\circ} \mathrm{C}$. Supernatants were collected after 96 hours, centrifuged, and stored at $-80{ }^{\circ} \mathrm{C}$ until use. A multiplex bead-based platform (Life Technologies) was performed on supernatants to measure the cytokine levels of human IFN- $\gamma$, IL-10, IL4, IL-5, IL-2, IL-9, IL-13, and IL-17 using the Human Th1/Th2/Th17 Magnetic 8-Plex Panel Kit according to the manufacturer's instructions. The use of Malian human samples was approved by Institutional Review Boards (IRBs) of the NIAID (clinical protocol NCT00344084) and by the ethics committee of the Faculty of Medicine, Pharmacy and Odontostomatology at the University of Bamako, Mali. All clinical investigations have been approved by the author's institution and conducted according to the Declaration of Helsinki principles, and written informed consent was obtained from all subjects. Human PBMCs from healthy subjects were obtained under written informed consent, from the NIH Clinical Center IRB-approved protocols from the NIH Clinical Center Department of Transfusion Medicine.

\section{Statistical analyses}

Lines present in the scatterplot graphs represent the mean, and bar graphs depict means \pm SEM or SD as indicated. Correlation graphs are presented with a linear regression slope and 95\% CI (dotted line). Statistical differences between two groups were tested by $t$ test (two-tailed Mann-Whitney test). One-way ANOVA followed by Fisher's least significant difference post-test was performed to analyze multiple groups. When the samples were paired, one-way ANOVA was followed by Dunn's multiple comparisons post-test. Correlations were tested by Spearman test, and dotted lines illustrate the $95 \%$ CI. Survival curves were analyzed by the log-rank (Mantel-Cox) test. We tested the distribution normality of the samples by the Shapiro-Wilk test. We considered $P<0.05$ as statistically significant. All statistical analyses were performed using the GraphPad Prism software.

\section{SUPPLEMENTARY MATERIALS}

www.sciencetranslationalmedicine.org/cgi/content/full/7/290/290ra90/DC1

Fig. S1. Disease progression of CL in NHP after transmission of $L$. major by bites of 50 infected P. duboscqi sand flies.

Fig. S2. $\mathrm{CD}^{+}$lymphocytes are the main source of Leishmania-specific IFN- $\gamma$ in NHP exposed to uninfected $P$. duboscqi sand flies.

Fig. S3. CD8 ${ }^{+}$lymphocytes are not critical for protection from $\mathrm{CL}$ in NHP exposed to uninfected sand fly bites. Fig. S4. Anti-PdSP15 antibodies and CD8 ${ }^{+}$lymphocytes are not critical for protection from $\mathrm{CL}$ in PdSP15-immunized NHP.

Fig. S5. The crystal structure of PdSP15.

Table S1. Measurements $(\mathrm{mm})$ of skin indurations 48 hours after inoculation with plasmids coding for selected sand fly salivary proteins.

Table S2. IFN- $\gamma$ and IL-4 mRNA expression by RT-qPCR.

Table S3. Data collection and refinement statistics for PdSP15.

Source data. Excel file

\section{REFERENCES AND NOTES}

1. J. Schroeder, T. Aebischer, Vaccines for leishmaniasis: From proteome to vaccine candidates. Hum. Vaccin. 7, 10-15 (2011).

2. S. Kamhawi, Y. Belkaid, G. Modi, E. Rowton, D. Sacks, Protection against cutaneous leish maniasis resulting from bites of uninfected sand flies. Science 290, 1351-1354 (2000).

3. J. G. Valenzuela, Y. Belkaid, M. K. Garfield, S. Mendez, S. Kamhawi, E. D. Rowton, D. L. Sacks, J. M. Ribeiro, Toward a defined anti-Leishmania vaccine targeting vector antigens: Characterization of a protective salivary protein. J. Exp. Med. 194, 331-342 (2001). 
4. R. Gomes, F. Oliveira, C. Teixeira, C. Meneses, D. C. Gilmore, D. E. Elnaiem, S. Kamhawi, J. G. Valenzuela Immunity to sand fly salivary protein LM11 modulates host response to vector-transmitted Leishmania conferring ulcer-free protection. J. Invest. Dermatol. 132, 2735-2743 (2012).

5. R. Gomes, C. Teixeira, M. J. Teixeira, F. Oliveira, M. J. Menezes, C. Silva, C. I. de Oliveira, J. C. Miranda, D. E. Elnaiem, S. Kamhawi, J. G. Valenzuela, C. I. Brodskyn, Immunity to a salivary protein of a sand fly vector protects against the fatal outcome of visceral leishmaniasis in a hamster model. Proc. Natl. Acad. Sci. U.S.A. 105, 7845-7850 (2008).

6. F. Oliveira, P. G. Lawyer, S. Kamhawi, J. G. Valenzuela, Immunity to distinct sand fly salivary proteins primes the anti-Leishmania immune response towards protection or exacerbation of disease. PLOS Negl. Trop. Dis. 2, e226 (2008).

7. M. E. Rogers, T. Ilg, A. V. Nikolaev, M. A. Ferguson, P. A. Bates, Transmission of cutaneous leishmaniasis by sand flies is enhanced by regurgitation of fPPG. Nature 430,463-467 (2004).

8. N. C. Peters, N. Kimblin, N. Secundino, S. Kamhawi, P. Lawyer, D. L. Sacks, Vector transmission of Leishmania abrogates vaccine-induced protective immunity. PLOS Pathog. 5, e1000484 (2009).

9. World Health Organization, Leishmaniasis, Fact sheet N³75, 2014; http://www.who.int/ mediacentre/factsheets/fs375/en/.

10. P. D. Ready, Biology of phlebotomine sand flies as vectors of disease agents. Annu. Rev. Entomol. 58, 227-250 (2013).

11. R. Reithinger, J. C. Dujardin, H. Louzir, C. Pirmez, B. Alexander, S. Brooker, Cutaneous leishmaniasis. Lancet Infect. Dis. 7, 581-596 (2007).

12. L. Holm, P. Rosenström, Dali server: Conservation mapping in 3D. Nucleic Acids Res. 38 W545-W549 (2010).

13. F. Oliveira, B. Traoré, R. Gomes, O. Faye, D. C. Gilmore, S. Keita, P. Traoré, C. Teixeira, C. A. Coulibaly, S. Samake, C. Meneses, I. Sissoko, R. M. Fairhurst, M. P. Fay, J. M. Anderson, S. Doumbia S. Kamhawi, J. G. Valenzuela, Delayed-type hypersensitivity to sand fly saliva in humans from a leishmaniasis-endemic area of Mali is $\mathrm{T}_{\mathrm{H}} 1$-mediated and persists to midlife. J. Invest. Dermatol. 133, 452-459 (2013).

14. F. Oliveira, S. Kamhawi, A. E. Seitz, V. M. Pham, P. M. Guigal, L. Fischer, J. Ward, J. G. Valenzuela, From transcriptome to immunome: Identification of DTH inducing proteins from a Phlebotomus ariasi salivary gland cDNA library. Vaccine 24, 374-390 (2006).

15. R. Gomes, F. Oliveira, The immune response to sand fly salivary proteins and its influence on leishmania immunity. Front. Immunol. 3, 110 (2012).

16. M. Abdeladhim, S. Kamhawi, J. G. Valenzuela, What's behind a sand fly bite? The profound effect of sand fly saliva on host hemostasis, inflammation and immunity. Infect. Genet. Evol. 28, 691-703 (2014).

17. N. Saljoughian, T. Taheri, F. Zahedifard, Y. Taslimi, F. Doustdari, A. Bolhassani, D. Doroud, H. Azizi, K. Heidari, M. Vasei, N. Namvar Asl, B. Papadopoulou, S. Rafati, Development of novel primeboost strategies based on a tri-gene fusion recombinant $L$. tarentolae vaccine against experimental murine visceral leishmaniasis. PLOS Negl. Trop. Dis. 7, e2174 (2013).

18. F. Zahedifard, E. Gholami, T. Taheri, Y. Taslimi, F. Doustdari, N. Seyed, F. Torkashvand, C. Meneses, B. Papadopoulou, S. Kamhawi, J. G. Valenzuela, S. Rafati, Enhanced protective efficacy of nonpathogenic recombinant Leishmania tarentolae expressing cysteine proteinases combined with a sand fly salivary antigen. PLOS Negl. Trop. Dis. 8, e2751 (2014).

19. M. E. Rogers, O. V. Sizova, M. A. Ferguson, A. V. Nikolaev, P. A. Bates, Synthetic glycovaccine protects against the bite of Leishmania-infected sand flies. J. Infect. Dis. 194, 512-518 (2006).

20. R. Gomes, C. Teixeira, F. Oliveira, P. G. Lawyer, D. E. Elnaiem, C. Meneses, Y. Goto, A. Bhatia R. F. Howard, S. G. Reed, J. G. Valenzuela, S. Kamhawi, KSAC, a defined Leishmania antigen, plus adjuvant protects against the virulence of $L$. major transmitted by its natural vector Phlebotomus duboscqi. PLOS Negl. Trop. Dis. 6, e1610 (2012).

21. T. Lestinova, M. Vlkova, J. Votypka, P. Volf, I. Rohousova, Phlebotomus papatasi exposure cross-protects mice against Leishmania major co-inoculated with Phlebotomus duboscai salivary gland homogenate. Acta Trop. 144, 9-18 (2015).

22. M. Abdeladhim, A. M. Ben, S. Marzouki, H. N. Belhadj, T. Boussoffara, H. N. Belhaj, S. A. Ben, $\mathrm{H}$. Louzir, Human cellular immune response to the saliva of Phlebotomus papatasi is mediated by IL-10-producing CD8+ T cells and Th1-polarized CD4+ lymphocytes. PLOS Negl. Trop. Dis. 5, e1345 (2011)

23. S. Marzouki, M. Abdeladhim, C. B. Abdessalem, F. Oliveira, B. Ferjani, D. Gilmore, H. Louzir J. G. Valenzuela, A. M. Ben, Salivary antigen SP32 is the immunodominant target of the antibody response to Phlebotomus papatasi bites in humans. PLOS Negl. Trop. Dis. 6 e1911 (2012).

24. E. Hajjar, D. Perahia, H. Debat, C. Nespoulous, C. H. Robert, Odorant binding and conformational dynamics in the odorant-binding protein. J. Biol. Chem. 281, 29929-29937 (2006).

25. P. H. Alvarenga, I. M. Francischetti, E. Calvo, A. Sá-Nunes, J. M. Ribeiro, J. F. Andersen, The function and three-dimensional structure of a thromboxane $A_{2}$ /cysteinyl leukotrienebinding protein from the saliva of a mosquito vector of the malaria parasite. PLOS Biol. 8, e1000547 (2010).

26. N. Collin, R. Gomes, C. Teixeira, L. Cheng, A. Laughinghouse, J. M. Ward, D. E. Elnaiem, L. Fischer, J. G. Valenzuela, S. Kamhawi, Sand fly salivary proteins induce strong cellular im- munity in a natural reservoir of visceral leishmaniasis with adverse consequences for Leishmania. PLOS Pathog. 5, e1000441 (2009).

27. K. Jain, N. K. Jain, Vaccines for visceral leishmaniasis: A review. J. Immunol. Methods, 10.1016/j.jim.2015.03.017 (2015).

28. R. Kumar, C. Engwerda, Vaccines to prevent leishmaniasis. Clin. Transl. Immunol. 3, e13 (2014).

29. V. S. Raman, M. S. Duthie, C. B. Fox, G. Matlashewski, S. G. Reed, Adjuvants for Leishmania vaccines: From models to clinical application. Front. Immunol. 3, 144 (2012).

30. N. Kimblin, N. Peters, A. Debrabant, N. Secundino, J. Egen, P. Lawyer, M. P. Fay, S. Kamhawi, D. Sacks, Quantification of the infectious dose of Leishmania major transmitted to the skin by single sand flies. Proc. Natl. Acad. Sci. U.S.A. 105, 10125-10130 (2008).

31. C. Maia, V. Seblova, J. Sadlova, J. Votypka, P. Volf, Experimental transmission of Leishmania infantum by two major vectors: A comparison between a viscerotropic and a dermotropic strain. PLOS Negl. Trop. Dis. 5, e1181 (2011).

32. N. F. Secundino, V. C. de Freitas, C. C. Monteiro, A. C. Pires, B. A. David, P. F. Pimenta, The transmission of Leishmania infantum chagasi by the bite of the Lutzomyia longipalpis to two different vertebrates. Parasit. Vectors 5, 20 (2012).

33. R. J. Probst, B. T. Wellde, P. G. Lawyer, J. S. Stiteler, E. D. Rowton, Rhesus monkey model for Leishmania major transmitted by Phlebotomus papatasi sandfly bites. Med. Vet. Entomol. 15, 12-21 (2001).

34. V. Vinhas, B. B. Andrade, F. Paes, A. Bomura, J. Clarencio, J. C. Miranda, A. Báfica, A. Barral, M. Barral-Netto, Human anti-saliva immune response following experimental exposure to the visceral leishmaniasis vector, Lutzomyia longipalpis. Eur. J. Immunol. 37, 3111-3121 (2007).

35. J. M. Anderson, S. Samake, G. Jaramillo-Gutierrez, I. Sissoko, C. A. Coulibaly, B. Traoré, C. Soucko, B. Guindo, D. Diarra, M. P. Fay, P. G. Lawyer, S. Doumbia, J. G. Valenzuela, S. Kamhawi, Seasonality and prevalence of Leishmania major infection in Phlebotomus duboscqi Neveu-Lemaire from two neighboring villages in central Mali. PLOS Negl. Trop. Dis. 5, e1139 (2011).

36. P. A. Darrah, D. T. Patel, P. M. De Luca, R. W. Lindsay, D. F. Davey, B. J. Flynn, S. T. Hoff, P. Andersen, S. G. Reed, S. L. Morris, M. Roederer, R. A. Seder, Multifunctional $\mathrm{T}_{\mathrm{H}} 1$ cells define a correlate of vaccine-mediated protection against Leishmania major. Nat. Med. 13, 843-850 (2007).

37. M. L. Precopio, M. R. Betts, J. Parrino, D. A. Price, E. Gostick, D. R. Ambrozak, T. E. Asher, D. C. Douek A. Harari, G. Pantaleo, R. Bailer, B. S. Graham, M. Roederer, R. A. Koup, Immunization with vaccinia virus induces polyfunctional and phenotypically distinctive $C D 8^{+} T$ cell responses. J. Exp. Med. 204 1405-1416 (2007).

38. S.-H. Park, E.-C. Shin, S. Capone, L. Caggiari, V. De Re, A. Nicosia, A. Folgori, B. Rehermann Successful vaccination induces multifunctional memory T-cell precursors associated with early control of hepatitis C virus. Gastroenterology 143, 1048-1060.e4 (2012).

39. E. K. Forbes, C. Sander, E. O. Ronan, H. McShane, A. V. Hill, P. C. Beverley, E. Z. Tchilian, Multifunctional, high-level cytokine-producing Th1 cells in the lung, but not spleen, correlate with protection against Mycobacterium tuberculosis aerosol challenge in mice. J. Immunol. 181, 4955-4964 (2008).

40. S. C. Derrick, I. M. Yabe, A. Yang, S. L. Morris, Vaccine-induced anti-tuberculosis protective immunity in mice correlates with the magnitude and quality of multifunctional CD4 T cells. Vaccine 29, 2902-2909 (2011).

41. M. R. Betts, M. C. Nason, S. M. West, S. C. De Rosa, S. A. Migueles, J. Abraham, M. M. Lederman J. M. Benito, P. A. Goepfert, M. Connors, M. Roederer, R. A. Koup, HIV nonprogressors preferentially maintain highly functional HIV-specific CD8 ${ }^{+}$T cells. Blood 107, 4781-4789 (2006).

42. R. A. Seder, P. A. Darrah, M. Roederer, T-cell quality in memory and protection: Implications for vaccine design. Nat. Rev. Immunol. 8, 247-258 (2008).

43. W. R. Faber, L. Oskam, T. van Gool, N. C. Kroon, K. J. Knegt-Junk, H. Hofwegen, A. C. van der Wal, P. A. Kager, Value of diagnostic techniques for cutaneous leishmaniasis. J. Am. Acad. Dermatol. 49, 70-74 (2003)

44. A. Sassi, H. Louzir, S. A. Ben, M. Mokni, O. A. Ben, K. Dellagi, Leishmanin skin test lymphoproliferative responses and cytokine production after symptomatic or asymptomatic Leish mania major infection in Tunisia. Clin. Exp. Immunol. 116, 127-132 (1999).

45. R. Alvarado, C. Enk, K. Jaber, L. Schnur, S. Frankenburg, Delayed-type hypersensitivity and lymphocyte proliferation in response to Leishmania major infection in a group of children in Jericho. Trans. R. Soc. Trop. Med. Hyg. 83, 189-192 (1989).

46. E. A. Khalil, H. AM El, E. E. Zijlstra, M. M. Mukhtar, H. W. Ghalib, B. Musa, M. E. Ibrahim, A. A. Kamil, M. Elsheikh, A. Babiker, F. Modabber, Autoclaved Leishmania major vaccine for prevention of visceral leishmaniasis: A randomised, double-blind, BCG-controlled trial in Sudan. Lancet 356 1565-1569 (2000).

47. M. Castés, J. Blackwell, D. Trujillo, S. Formica, M. Cabrera, G. Zorrilla, A. Rodas, P. L. Castellanos, J. Convit, Immune response in healthy volunteers vaccinated with killed leishmanial promastigotes plus BCG. I: Skin-test reactivity, T-cell proliferation and interferon- $\gamma$ production. Vaccine 12, 1041-1051 (1994).

48. W. Mayrink, C. A. da Costa, P. A. Magalhães, M. N. Melo, M. Dias, A. O. Lima, M. S. Michalick, P. Williams, A field trial of a vaccine against American dermal leishmaniasis. Trans. $R$. Soc. Trop. Med. Hyg. 73, 385-387 (1979). 
49. S. A. Ben, H. Louzir, S. Chlif, M. Mokni, A. Zaatour, M. Raouene, R. B. Ismail, K. Dellagi, The predictive validity of naturally acquired delayed-type hypersensitivity to leishmanin in resistance to Leishmania major-associated cutaneous leishmaniasis. J. Infect. Dis. 192, 1981-1987 (2005).

50. O. Bacellar, D. Faria, M. Nascimento, T. M. Cardoso, K. J. Gollob, W. O. Dutra, P. Scott, E. M. Carvalho, Interleukin 17 production among patients with American cutaneous leishmaniasis. J. Infect. Dis. 200, 75-78 (2009)

51. S. Brosch, K. Dietze-Schwonberg, S. Lopez Kostka, B. Lorenz, S. Haak, B. Becher, E. von Stebut, Disease control in cutaneous leishmaniasis is independent of IL-22. J. Invest. Dermatol. 135, 308-311 (2015).

52. N. S. Geraci, R. M. Mukbel, M. T. Kemp, M. N. Wadsworth, E. Lesho, G. M. Stayback, M. M. Champion, M. A. Bernard, M. Abo-Shehada, I. V. Coutinho-Abreu, M. Ramalho-Ortigão, H. A. Hanafi, E. Y. Fawaz, S. S. El-Hossary, G. Wortmann, D. F. Hoel, M. A. McDowell, Profiling of human acquired immunity against the salivary proteins of Phlebotomus papatasi reveals clusters of differential immunoreactivity. Am. J. Trop. Med. Hyg. 90, 923-938 (2014).

53. S. Nakae, Y. Komiyama, A. Nambu, K. Sudo, M. Iwase, I. Homma, K. Sekikawa, M. Asano, Y. Iwakura, Antigen-specific T cell sensitization is impaired in IL-17-deficient mice, causing suppression of allergic cellular and humoral responses. Immunity 17, 375-387 (2002).

54. M. B. Teunissen, C. W. Koomen, R. de Waal Malefyt, E. A. Wierenga, J. D. Bos, Interleukin-17 and interferon- $\gamma$ synergize in the enhancement of proinflammatory cytokine production by human keratinocytes. J. Invest. Dermatol. 111, 645-649 (1998).

55. P. Probst, E. Stromberg, H. W. Ghalib, M. Mozel, R. Badaro, S. G. Reed, J. R. Webb, Identification and characterization of T cell-stimulating antigens from Leishmania by CD4 T cell expression cloning. J. Immunol. 166, 498-505 (2001).

56. P. H. Alvarenga, X. Xu, F. Oliveira, A. C. Chagas, C. R. Nascimento, I. M. Francischetti, M. A. Juliano, L. Juliano, J. Scharfstein, J. G. Valenzuela, J. M. Ribeiro, J. F. Andersen, Novel family of insect salivary inhibitors blocks contact pathway activation by binding to polyphosphate, heparin and dextran sulfate. Arterioscler. Thromb. Vasc. Biol. 33, 2759-2770 (2013).

57. A. J. McCoy, R. W. Grosse-Kunstleve, P. D. Adams, M. D. Winn, L. C. Storoni, R. J. Read, Phaser crystallographic software. J. Appl. Crystallogr. 40, 658-674 (2007).

58. J. E. Sokal, Editorial: Measurement of delayed skin-test responses. N. Engl. J. Med. 293, 501-502 (1975).

59. L. Nicolas, E. Prina, T. Lang, G. Milon, Real-time PCR for detection and quantitation of Leishmania in mouse tissues. J. Clin. Microbiol. 40, 1666-1669 (2002).
Acknowledgments: We thank $E$. Morales and C. Rhodes for their outstanding technical assistance in rearing and providing sand flies. We thank R. Howard from the Infectious Diseases Research Institute (Seattle) for providing the adjuvant GLA-SE. We thank the animal facility personnel at Walter Reed Army Institute of Research for their outstanding technical and animal care assistance. Funding: Support for this work was provided by the Intramural Research Program at the NIAID, NIH, and by the Grand Challenges Explorations grant from the Bill and Melinda Gates Foundation. A.B.G.C. was partially funded by a Science Without Borders Fellowship from the National Council for Scientific and Technological Development (CNPq)-Brazil. Fundação de Amparo à Pesquisa do Rio de Janeiro Carlos Chagas Filho and Conselho Nacional de Desenvolvimento Científico e Tecnológico (P.H.A.). Author contributions: F.O. participated in all laboratory, animal, and human studies, performed statistical analysis, designed experiments, and drafted the manuscript. E.R. participated in all animal studies and designed experiments. R.G., C.T., and P.A.C. participated in laboratory and animal studies. M.A. participated in laboratory and human studies. P.G.L., H.A., and T.E.R. participated in animal studies. L.T.K., A.B.G.-C., and D.G. participated in laboratory studies. C.M. participated in rearing and providing sand flies for the study. P.H.A. and J.F.A. participated in expression of recombinant protein and solving the structure of the recombinant protein. S.D. participated in human studies. S.G.R. participated in adjuvant formulation and delivery. The project was scientifically conceived and directed by S.K. and J.G.V.; F.O., S.K., and J.G.V. wrote the manuscript. All authors took part in the critical review of the manuscript. Competing interests: The authors declare that they have no competing financial interests. Data and materials availability: The structure and coordinates are available at the RCSB PDB with PDB code 4OZD. Recombinant PdSP15 is available upon request after a material transfer agreement with NIAID office of technology transfer. The opinions or assertions contained herein are the private views of the author and are not to be construed as official or as reflecting true views of the Department of the Army or the Department of Defense.

Submitted 4 February 2015

Accepted 7 May 2015

Published 3 June 2015

10.1126/scitranslmed.aaa3043

Citation: F. Oliveira, E. Rowton, H. Aslan, R. Gomes, P. A. Castrovinci, P. H. Alvarenga, M. Abdeladhim, C. Teixeira, C. Meneses, L. T. Kleeman, A. B. Guimarães-Costa, T. E. Rowland, D. Gilmore, S. Doumbia, S. G. Reed, P. G. Lawyer, J. F. Andersen, S. Kamhawi, J. G. Valenzuela, A sand fly salivary protein vaccine shows efficacy against vector-transmitted cutaneous leishmaniasis in nonhuman primates. Sci. Transl. Med. 7, 290 ra90 (2015). 


\author{
A sand fly salivary protein vaccine shows efficacy against \\ vector-transmitted cutaneous leishmaniasis in nonhuman primates \\ Fabiano Oliveira et al. \\ Sci Transl Med 7, 290ra90 (2015); \\ DOI: 10.1126/scitransImed.aaa3043
}

Editor's Summary

\title{
Leishmania vaccine targets the messenger
}

The old adage "Don't kill the messenger" may not hold true when vaccinating against leishmaniasis. Oliveira et al. demonstrate that a vaccine against sand fly salivary protein can protect nonhuman primate from leishmania infection. Leishmaniasis is transmitted by the bite of infected phlebotomine sand flies, which also transfer some of their saliva with the bite. Most macaques vaccinated against PdSP15, a sand fly salivary protein, had decreased parasite burden after induction of cutaneous leishmaniasis initiated by infected bites. Moreover, people exposed to sand fly bites developed an immune response to PdSP15 as well, suggesting that this approach may be translatable to humans.

A complete electronic version of this article and other services, including high-resolution figures, can be found at:

/content/7/290/290ra90.full.html

Supplementary Material can be found in the online version of this article at: /content/suppl/2015/06/01/7.290.290ra90.DC1.html

Related Resources for this article can be found online at:

http://stm.sciencemag.org/content/scitransmed/5/202/202ra121.full.html

http://stm.sciencemag.org/content/scitransmed/6/234/234ra56.full.html

Information about obtaining reprints of this article or about obtaining permission to reproduce this article in whole or in part can be found at:

http://www.sciencemag.org/about/permissions.dtl 